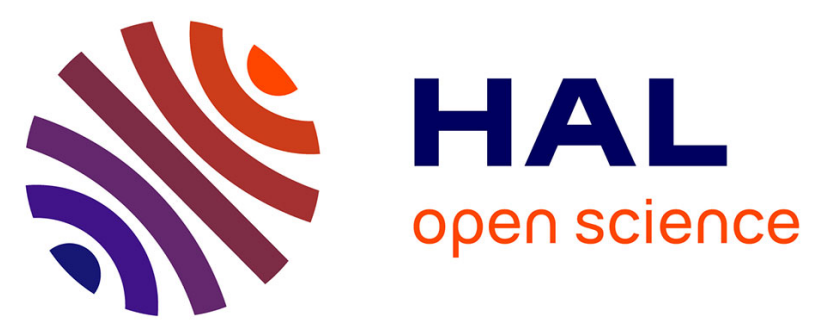

\title{
High Frequency Quantitative Ultrasound Spectroscopy of Excised Canine Livers and Mouse Tumors using the Structure Factor Model
}

Pauline Muleki-Seya, Régine Guillermin, Julien Guglielmi, Jiangang Chen, Thierry Pourcher, Elisa Konofagou, Emilie Franceschini

\section{To cite this version:}

Pauline Muleki-Seya, Régine Guillermin, Julien Guglielmi, Jiangang Chen, Thierry Pourcher, et al.. High Frequency Quantitative Ultrasound Spectroscopy of Excised Canine Livers and Mouse Tumors using the Structure Factor Model. IEEE Transactions on Ultrasonics, Ferroelectrics and Frequency Control, 2016, 10.1109/TUFFC.2016.2563169 . hal-01312679

\section{HAL Id: hal-01312679 \\ https://hal.science/hal-01312679}

Submitted on 8 May 2016

HAL is a multi-disciplinary open access archive for the deposit and dissemination of scientific research documents, whether they are published or not. The documents may come from teaching and research institutions in France or abroad, or from public or private research centers.
L'archive ouverte pluridisciplinaire HAL, est destinée au dépôt et à la diffusion de documents scientifiques de niveau recherche, publiés ou non, émanant des établissements d'enseignement et de recherche français ou étrangers, des laboratoires publics ou privés. 


\title{
High Frequency Quantitative Ultrasound
}

\section{Spectroscopy of Excised Canine Livers and Mouse Tumors using the Structure Factor Model}

\author{
Pauline Muleki-Seya, Régine Guillermin, Julien Guglielmi, Jiangang Chen, Thierry Pourcher, Elisa \\ Konofagou, Member IEEE, Emilie Franceschini, Member IEEE,
}

\begin{abstract}
Three scattering models were examined for characterizing ex vivo canine livers and HT29 mouse tumors in the 10-38 MHz and the 15-42 MHz frequency bandwidth, respectively. The Spherical Gaussian Model (SGM) and the Fluid Sphere Model (FSM) that were examined are suitable for dealing with sparse media, whereas the Structure Factor Model (SFM) is adapted for characterizing concentrated media. For the canine livers, the scatterer radius and acoustic concentration estimated with the three models were similar and matched well the nuclear structures obtained from histological analysis (with relative errors less than 7\%). These results show that the livers could be considered as a diluted medium and that the nuclei in liver could be a dominant source of scattering. For the homogeneous mouse tumors, containing mostly viable HT29 cells, scatterer radius and volume fraction estimated with the SFM showed good agreement with the whole cell structures obtained from histological analysis (with relative errors less than 15\%), whereas the sparse models (SGM and FSM) gave no consistent quantitative ultrasound parameters. This suggests that the viable HT29 cell areas have densely packed cellular content and that the whole HT29 cell could be responsible for scattering. For the heterogeneous tumors, the hyperechogenic zones observed in the B-mode images were linked to the presence of small necrotic areas surrounded by viable HT29 cells. Comparison between sparse and concentrated models shows that these hyperechogenic zones could be considered as a concentrated medium.
\end{abstract}

This work has been carried out thanks to the support of the Canceropole PACA and the A*MIDEX project (ANR-11-IDEX-0001-02) funded by the "Investissements d'Avenir" French Government program, managed by the French National Research Agency (ANR).

Pauline Muleki-Seya, Régine Guillermin and Emilie Franceschini are with Laboratoire de Mécanique et d'Acoustique LMA - CNRS UPR 7051, Aix-Marseille University, Centrale Marseille, Marseille, France (e-mail: franceschini@lma.cnrs-mrs.fr).

Julien Guglielmi and Thierry Pourcher are with Laboratoire TIRO, UMRE 4320, iBEB, DSV, CEA, University of Nice Sophia Antipolis, Nice, France.

Jiangang Chen and Elisa Konofagou are with Department of Biomedical Engineering and Radiology, Columbia University, New York, NY 10027 USA. 


\section{High Frequency Quantitative Ultrasound}

\section{Spectroscopy of Excised Canine Livers and Mouse Tumors using the Structure Factor Model}

\section{INTRODUCTION}

Quantitative ultrasound (QUS) techniques providing insight into tissue microstructure are based on the frequencybased analysis of the signals backscattered from biological tissues. A spectral analysis approach consists in estimating two spectral parameters: spectral slope and intercept [1]. The spectral slope is the slope with respect to the frequency of the linear regression of the normalized power spectrum (expressed in $\mathrm{dB}$ ) and the spectral intercept is the extrapolation of the normalized power spectrum linear fit to zero frequency. The slope is related to the effective scatterer radius, and the intercept is determined by the scatterer radius and acoustic concentration (the acoustic concentration is the product of the scatterer volume fraction times the square of the relative impedance difference between the scatterers and surrounding tissue). Another QUS method used to extract effective scatterer radius and acoustic concentration relies on theoretical scattering models in order to fit the measured backscatter coefficient (BSC) from biological tissues to an estimated BSC using an appropriate theoretical scattering model [2]. Generally, two models are used to describe the scattering from tissue. The more frequently used model, called the spherical Gaussian model (SGM), describes tissue as a random inhomogeneous continuum with impedance fluctuations [1], [3]. The second model, called the Fluid Sphere Model (FSM), describes tissue as an ensemble of discrete scatterers with an impedance differing from that of a homogeneous background medium, where the cells are generally considered as the dominant source of scatterers and modeled as fluid spheres [4]. Both models yield two QUS parameters: the average scatterer radius and the acoustic concentration.

The goal of QUS is to associate these parameter values with specific tissue structures [1]. Feleppa et al. demonstrated that the effective scatterer radius was relevant to detect ocular [5] or prostate tumors [6]. In ocular malignant tumors, larger scatterer radii were estimated by ultrasound when compared to normal tissues and were related to clusters of melanin-laden histiocytes [5]. Waag et al. related the ultrasound backscatter measurements to the lobule structures in the liver [7]. Oelze et al. distinguished fibroadenomas (benign tumors) and carcinomas (malignant tumors) based on the QUS estimates [8]. The scatterer radius QUS images suggest that fibroadenomas have larger scatterers consistent with the glandular acini radii and that carcinomas have smaller, more uniform scatterer radii [8]. However, it is often difficult to establish a relationship between QUS scatterer property estimates and actual tissue structures (generally identified from optical microscope images) [4], [9]. Oelze and O’Brien compared three scattering models (SGM, FSM and a new cell model to consider backscattering from cell nuclei 
and cytoskeleton) to examine two mouse models of mammary cancers: carcinomas containing uniformly distributed cells and sarcomas containing cell clusters [4]. None of the three models produced good fits to the data, and QUS estimates did not faithfully represent the actual microstructural differences as observed from histological images [4].

In the aforementioned models (SGM, FSM and new cell model), the scatterers are assumed to be independently and randomly distributed (i.e., to have a low scatterer volume fraction) and multiple scattering is neglected (in line with the Born approximation). Under these hypotheses, the power of the backscattered signals increases linearly with the scatterer volume fraction and depends on the radius and acoustic properties of the scattering structures. This linear relationship has been used to monitor the scatterer radius and/or acoustic concentration. However, the assumption of randomly distributed scatterers may not hold in concentrated cell pellet biophantoms [11] or in tumors with densely packed cells [12]. Therefore, our hypothesis is that a scattering model considering the interference effects caused by the correlations among scatterer positions (i.e. considering the coherent scattering) may help to estimate QUS parameters with better accuracy on densely-packed media. The Structure Factor Model (SFM) is based on the same hypothesis used in the FSM that the cells are a dominant source of scattering. The SFM considers the coherent scattering by summing the contributions from individual cells and modeling the cellular interactions by a statistical mechanics structure factor [13], [14]. Our group performed experiments on tissue-mimicking phantoms [15] and cell pellet biophantoms [16] to show the superiority of the SFM in comparison with other classical models that do not take the structure factor into account (i.e., the SGM and the FSM) to explain the BSC behavior for concentrated media. More recently, the SGM, the FSM and the SFM were compared to estimate the scatterer radius and the acoustic concentration from backscattering measurements for cell pellet biophantoms [17]. In that study, the three scattering models gave similar QUS parameters for diluted media $\left(\phi^{*} \leq 0.06\right)$. However, the SGM and the FSM did not yield accurate structural parameter estimates for the highest volume fractions $\phi^{*} \geq 0.12$, whereas the SFM yielded satisfactory estimates for actual cell volume fractions ranging from 0.006 to 0.30 , i.e. for both diluted and concentrated cell pellet biophantoms [17].

The present study examined the SFM to estimate QUS properties of excised canine livers and mouse tumors, and compared the use of the SFM with that of the SGM and the FSM. The canine liver is quite homogeneous whereas some of the mouse tumors were composed of two different areas: a cellular area containing viable tumor cells and a necrotic area containing lymphocytes and no viable tumor cells. The ability of the SFM to estimate simultaneously the scatterer radius, the volume fraction and the relative contrast impedance was evaluated. These QUS parameter estimates obtained from the three models were compared to the true cellular structure properties observed from histological slides.

\section{MATERiAls AND Methods}

\section{A. Excised tissues}

1) Canine livers: Experiments were conducted on four excised canine livers obtained from four mongrel male dogs. Liver was used because of its relative homogeneity compared with other tissue. Before ultrasound scanning, each specimen was immersed in Phosphate Buffered Saline (PBS) solution and placed in a vacuum chamber for 
30 min prior to each experiment. All animal experimental procedures were approved by the Columbia University Institutional Animal Care and Use Committee (protocol number: Columbia University AC-AAAD5650).

2) Mouse tumors: The human colon adenocarcinoma cell line HT29 was grown in Dulbecco's modified Eagles's medium containing $4.5 \mathrm{~g}$ of glucose/liter and supplemented with $10 \%$ fetal calf serum. This cell line represents a very useful xenograft model. HT29 xenografts grow rapidly and is a poor vascularized tissue. Necrotic areas could appear in the tumor core as a result of rapid growth and exacerbated by the low vascularity of this tumor model. $10^{6}$ HT29 cells in $100 \mu \mathrm{L}$ of PBS were subcutaneously inoculated into the flank of 6 to 8 -week-old Nude mice (Charles River Laboratories). After two or three weeks of growth, the mice were anesthetized and euthanized under anesthesia by cervical dislocation. For each mouse, the tumor was excised and a part of the skin was removed. The tumors were then immersed in PBS and were ultrasonically imaged. A total of six tumors were used in this study. Animal housing and procedures were conducted according to the guidelines of the French Agriculture Ministry and were approved by the local ethics committee.

The time between euthanasia and the end of ultrasonic acquisition was comprised between one hour and one hour and a half for all experiments, on both canine livers and mouse tumors.

\section{B. High frequency ultrasound data acquisition}

Ultrasound measurements were acquired using a Vevo 770 high frequency ultrasound system (Visualsonic Inc, Toronto, Canada) with a RMV 707 probe for the liver measurements and with a RMV 703 probe for the mouse tumor measurements. The oscillating single-element focused circular transducer of the RMV 707 and the RMV 703 probes had center frequencies of 25 and $30 \mathrm{MHz}$, focuses of 12.7 and $10 \mathrm{~mm}$, and f-numbers of 2.1 and 2.5 , respectively. The lateral resolution of both probes was approximately $115 \mu \mathrm{m}$. The radio frequency (RF) data were acquired from this scanner at a sampling frequency of $100 \mathrm{MHz}$ for the canine livers and $250 \mathrm{MHz}$ for the mouse tumors with 8 bit resolution using a data acquisition board (GaGe, USA).

RF data were collected from 20 different scan planes with a step size of $500 \mu \mathrm{m}$ for the livers, and from 5 to 10 different scan planes in the middle of the tumor with a step size of $300 \mu \mathrm{m}$ for the mouse tumors, using a micropositioning system. Note that, in the case of the liver, the orientation and position of the probe were chosen to prevent the presence in the scan plane of specular echoes originating from the veins or arteries. A scan plane contains approximately 312 echo lines. The probe focus was positioned between 1 and $2 \mathrm{~mm}$ below the PBS/tissue interface. For each scan plane, the data were separated into overlapping rectangular regions-of-interest (ROIs), defined as the gated region. The axial length of an ROI corresponds to the range-gated RF echo with length of $0.6 \mathrm{~mm}$ for the livers and of $0.5 \mathrm{~mm}$ for the tumors, corresponding to ten wavelengths at the center frequency. The lateral length of an ROI corresponds to 60 adjacent echo lines from the Visualsonics probe (corresponding to a length comprised between 0.6 and $1 \mathrm{~mm}$ ). BSC estimates were obtained for each ROI from the RF signals, as described below in section II-C. ROIs were chosen within the liver or within the tumors where the B-mode images appeared to be quite homogeneous. The overlap of ROIs was $75 \%$ for both lateral and axial directions. Note that only one depth was examined for the liver experiments since a large number of scan planes (equal to 20) was collected. The average number of independent QUS estimates for a single liver was 80 . For the mouse tumors, 
the scanned depth varied according to tumor volumes and ranged between 1.1 and $3.8 \mathrm{~mm}$ in the axial direction (corresponding to a number of overlapping ROIs comprised between 6 and 27, respectively).

\section{Attenuation and BSC measurement}

The attenuation coefficients of the tissues were determined by using a standard substitution method [18]. Experiments were conducted with a focused transducer with center frequency of $20 \mathrm{MHz}$, focus of $13 \mathrm{~mm}$ and f-number of 2.1. The power spectrum of the emitted signal, denoted $S_{r e f}(f)$, is found by reflecting the pulse from a reflector, with no tissue in the acoustic path. A tissue specimen is then put in contact with the reflector to determine its thickness as well as its attenuation. The thickness of the tissue specimen $D$ is deduced from the time of flight between the transducer and the PBS/tissue interface. The spectrum of the pulse transmitted through the tissue back to the transducer is denoted $S(f)$. The measured tissue attenuation $\alpha_{0}$ (in $\mathrm{dB} / \mathrm{mm}$ ) is computed by taking the $\log$ spectral difference between the two spectra [18]

$$
\alpha_{0}(f)=\alpha_{P B S}(f)+\frac{10}{2 D} \log _{10}\left(\frac{S_{r e f}(f)}{S(f)}\right)
$$

where $f$ is the frequency, $\alpha_{P B S}$ is the frequency-dependent attenuation of PBS, which is taken to be similar to water, $2.17 \times 10^{-4} \mathrm{~dB} \cdot \mathrm{MHz}^{-2} \cdot \mathrm{m}^{-1}$ at $20{ }^{\circ} \mathrm{C}$ [19]. Assuming that the tissue attenuation increases linearly with the frequency $\left(\alpha_{0}(f)=\alpha f\right)$, a linear regression analysis provides the attenuation coefficient $\alpha$ in $\mathrm{dB} / \mathrm{mm} / \mathrm{MHz}$. For each tissue specimen, the averaged attenuation coefficient was obtained from 10 different locations in the tissue specimen. The mean attenuation coefficients were $\alpha_{L}=0.65 \pm 0.05 \mathrm{~dB} / \mathrm{cm} / \mathrm{MHz}(n=4), \alpha_{T}=0.63 \pm 0.09 \mathrm{~dB} / \mathrm{cm} / \mathrm{MHz}$ ( $n=4)$ and $\alpha_{S}=3.36 \pm 0.06 \mathrm{~dB} / \mathrm{cm} / \mathrm{MHz}(n=2)$, for the canine liver, the HT29 mouse tumor and the skin, respectively ( $n$ being the number of tissue specimens measured). The attenuation coefficients found in the literature were in the same range: $0.69 \mathrm{~dB} / \mathrm{cm} / \mathrm{MHz}$ at $10-30 \mathrm{MHz}$ for the bovine liver [20], $0.6 \mathrm{~dB} / \mathrm{cm} / \mathrm{MHz}$ at $10-30 \mathrm{MHz}$ for the mouse carcinoma tumor [21] and 2.6 dB/cm/MHz at 11-26.6 MHz for the human dermis [22].

To take into account the attenuation effects, attenuation compensation was conducted individually for every RF line of each ROI. In the case of the canine liver experiments, sound has to travel through only one layer of liver tissue corresponding to the distance between the PBS/liver interface and the beginning of the ROI, denoted $d_{L}$. In the case of the mouse tumor experiments, before reaching the beginning of the ROI, sound had to pass through two layers of attenuating tissues: a first layer of highly-attenuating residual skin of length $d_{S}$ and a second layer composed mainly of HT29 cells of length $d_{T}$ (see Fig. 1). For each RF segment of the ROI, the values of $d_{S}$ and $d_{T}$ are different. Note that the residual skin boundaries were delineated manually. The power spectrum of the RF segment, denoted $P_{\text {meas }}$, was compensated for the attenuation effects as follows [23]

$$
\begin{aligned}
P_{\text {meas }}(f)= & \left|F T\left\{R F_{L}(t) W_{L}(t)\right\}\right|^{2} e^{4 d_{L} \alpha_{L}(f)} \\
= & {\left[\frac{4 L \alpha_{L}(f)}{1-e^{-4 L \alpha_{L}(f)}}\right] \text { for the liver } } \\
= & \left|F T\left\{R F_{L}(t) W_{L}(t)\right\}\right|^{2} e^{4 d_{S} \alpha_{S}(f)} \\
& e^{4 d_{T} \alpha_{T}(f)}\left[\frac{4 L \alpha_{T}(f)}{1-e^{-4 L \alpha_{T}(f)}}\right] \text { for the mouse tumor }
\end{aligned}
$$


where $F T$ is the Fourier transform operator, $R F_{L}$ is the raw RF-segment signal data and $W_{L}$ is the rectangular window of length $L$ centered on the middle of the RF segment. Finally, the 60 attenuation-compensated power spectra of the RF signals in the ROI were averaged to obtain $\overline{P_{\text {meas }}}$.

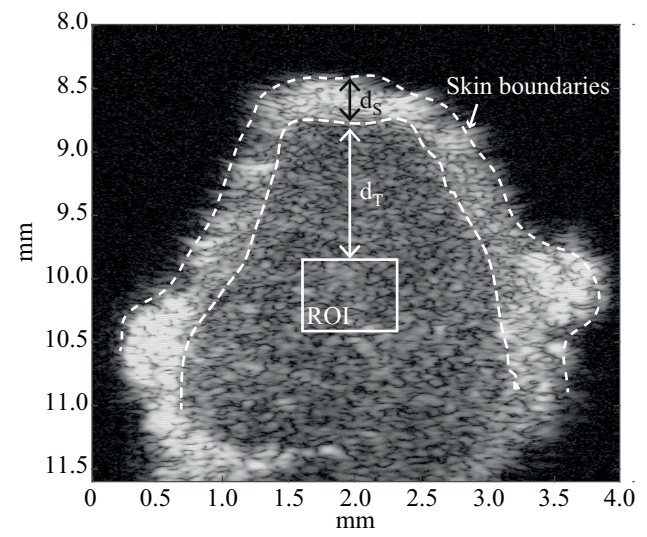

Fig. 1. Illustration of the data processing of a mouse tumor.

QUS images based on BSC analysis depict tissue properties in a system-independent manner. A reference phantom technique [24] was employed for BSC estimation to account for the electromechanical system response and the depth-dependent diffraction and focusing effects of the ultrasound beam. The reference phantom was composed of distilled water, $2 \%$ agar powder (A9799, Sigma Aldrich, France), and 1\% of polyamide microsphere with a radius of $2.5 \mu \mathrm{m}$ (orgasol 2001 UD NAT1, Arkema, France). Polyamide microspheres (sound speed of $2300 \mathrm{~m} / \mathrm{s}$, density of $1030 \mathrm{~kg} / \mathrm{m}^{3}$ and Poisson's ratio of 0.42) have been already used in studies of BSC estimation [15]. The reference sample is easy to prepare and to handle, and the scattering process occurring in an ensemble of identical solid microspheres at a very low volume fraction of $1 \%$ (dilute medium) has been well documented using the Faran model [15], [25]. Echoes from the reference scattering medium were acquired and windowed, as had previously been done with the tissue specimen under consideration. Their power spectra were compensated for the attenuation (using $\alpha_{r e f}=0.004 \mathrm{~dB} / \mathrm{mm} / \mathrm{MHz}$, which was measured independently by a standard substitution method [18]) and then averaged to obtain $\overline{P_{\text {ref }}}$. The measured BSC was thus computed as follows [24]

$$
B S C_{\text {meas }}(k)=B S C_{\text {ref }}(k) \frac{\overline{P_{\text {meas }}(k)}}{\overline{P_{\text {ref }}(k)}}
$$

where $k$ is the wavenumber and $B S C_{\text {ref }}$ is the theoretical BSC of the reference sample given by Eq. (2) in Ref. [15] using the Faran model [25].

\section{QUS parameter estimates}

The present study compared QUS estimates from three models - the SGM, the FSM and the SFM. Using the SGM, the BSC is modeled using a spatial autocorrelation function describing the size, shape, acoustic properties, and distribution of the scatterers in the medium. The BSC can be expressed as the product of the BSC in the Rayleigh limit and the backscatter form factor $F F$ [26]. The form factor describes the frequency dependence of 
the scattering, in terms of the size, shape, and acoustic properties of the scatterers. The Gaussian form factor has been used for many applications [1] - [4] and models a medium with continuous changes in acoustic properties. The theoretical BSC using the SGM formulation is given by [26]

$$
B S C_{\mathrm{SGM}}(k)=\frac{k^{4} V_{s}^{2} n_{z}}{4 \pi^{2}} e^{-2 k^{2} d^{2}}=\frac{k^{4} V_{s}^{2} n_{z}}{4 \pi^{2}} e^{-0.827 k^{2} a_{G}^{2}},
$$

where $n_{z}$ is the acoustic concentration, $d$ is the correlation distance that characterized the continuous isotropic medium and $V_{s}=\left(2 \pi d^{2}\right)^{3 / 2}$. The effective scatterer radius $a_{G}$ is related to the correlation distance $d$ by setting values of $V_{s}$ for a continuum model equal to the volume of an effective sphere of radius $a_{G}: V_{s}=\left(2 \pi d^{2}\right)^{3 / 2}=$ $(4 / 3) \pi a_{G}^{3}$ (see section IV in Ref. 26).

The FSM describes tissue as an ensemble of discrete scatterers with an impedance differing from that of a homogeneous background medium. By considering an ensemble of identical fluid spheres of radius $a_{F}$ (randomly and independently distributed), the theoretical BSC for the FSM formulation is given by [26]

$$
B S C_{\mathrm{FSM}}(k)=\frac{k^{4} V_{s}^{2} n_{z}}{4 \pi^{2}}\left[\frac{3}{\left(2 k a_{F}\right)^{3}} j_{1}\left(2 k a_{F}\right)\right]^{2},
$$

where $j_{1}$ is the spherical Bessel function of the first kind of order 1 , and $a_{F}$ is the radius of the fluid sphere. When using the SGM or the FSM, the unknown QUS parameters are the acoustic concentration $n_{z}$ and the effective scatterer radius $a_{G}$ for the SGM (or $a_{F}$ for the FSM). In the rest of the paper, the subscript $G$ or $F$ for the effective scatterer radius will be omittted.

The SFM is based on the assumption that, at high scatterer volume fractions, interference effects are mainly caused by correlations between the spatial positions of individual scatterers, that can be modeled with the structure factor. By considering an ensemble of identical spheres of radius $a$, the theoretical BSC for the SFM formulation is given by [14], [15]

$$
B S C_{\mathrm{SFM}}(k)=n \frac{k^{4} V_{s}^{2} \gamma_{z}^{2}}{4 \pi^{2}}\left[\frac{3}{(2 k a)^{3}} j_{1}(2 k a)\right]^{2} S(k)
$$

where $n$ is the sphere number density and $S$ is the structure factor, which can be obtained analytically as described in [15, Eq. (A1)-(A4)] based on [27]. The unknown QUS parameters are the scatterer radius $a$, the volume fraction $\phi$ and the relative impedance contrast $\gamma_{z}$.

Estimated values of the QUS parameters were determined by fitting the measured $B S C_{\text {meas }}$ to the theoretical BSCs, i.e., by minimizing the cost function:

$$
F=\sum_{j}\left\|B S C_{\text {meas }}\left(k_{j}\right)-B S C_{\text {theo }}\left(k_{j}\right)\right\|^{2},
$$

where the $B S C_{\text {theo }}$ is given by Eq. (3) for the SGM, by Eq. (4) for the FSM and by Eq. (5) for the SFM. The cost functions were minimized over 10-38 MHz for the canine liver experiments with the RMV 707 probe and over 15-42 MHz for the HT29 mouse tumor with the RMV 703 probe, corresponding approximately to the $-12 \mathrm{~dB}$ frequency bandwidth of $\overline{P_{\text {meas }}}$. For the three models, the fitting procedure was performed by using the minimization routine "fminsearch" without constraint in MATLAB (The MathWorks, Inc., Natick, MA), i.e., a Nelder-Mead simplex method. In the case of the SFM, the "fmincon" routine was also tested with the constraint conditions that $0 \leq a \leq 100$ $\mu \mathrm{m}, 0 \leq \phi \leq 1$ and $0 \leq \gamma_{z} \leq 0.20$. The "fmincon" and "fminsearch" routines were compared because "fminsearch" 
sometimes gave unrealistic values for the estimated relative impedance contrast $\gamma_{z}$ (up to values of 0.7). This last point will be discussed later in section IV-C. For the purpose of comparison between the sparse models and the SFM, the acoustic concentration for the SFM was also calculated as: $n_{z}^{*}=\frac{\phi^{*} \gamma_{z}^{* 2}}{(4 / 3) \pi a^{* 3}}$.

For the three scattering models (SGM, FSM and SFM), the goodness of fit of the model to the measured $B S C_{\text {meas }}$ was assessed by the goodness-of-fit statistic, $R^{2}$, given by [4]:

$$
R^{2}=1-\frac{\sum_{j}\left\|B S C_{\text {meas }}\left(k_{j}\right)-B S C_{\text {opt }}\left(k_{j}\right)\right\|^{2}}{\sum_{j}\left\|B S C_{\text {meas }}\left(k_{j}\right)-\overline{B S C_{\text {opt }}}\right\|^{2}}
$$

where $B S C_{\mathrm{opt}}$ represents the theoretical BSC computed with the estimated values of the QUS parameters (obtained by the minimization procedure), and $\overline{B S C_{\mathrm{opt}}}$ represents the mean value of $B S C_{\mathrm{opt}}$ over the studied frequency range (10-38 MHz for the canine livers and 15-42 MHz for the HT29 mouse tumors). The closer the $R^{2}$ value is to 1 , the better the fit of the model to the data.

(a)

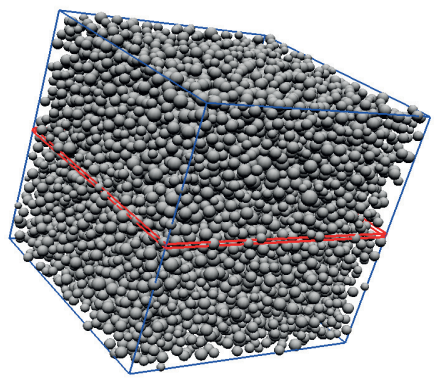

(b)

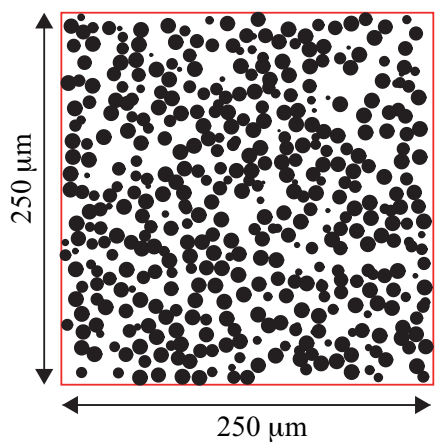

(c)

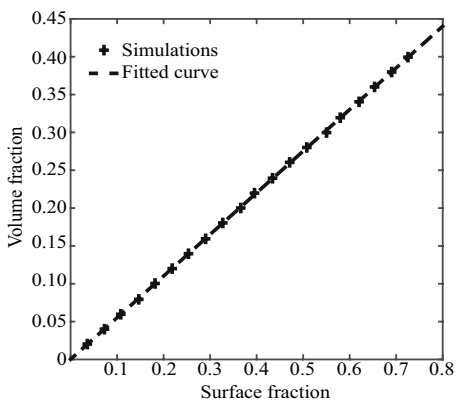

Fig. 2. (a) Three-dimensional illustration of a Gaussian distribution of spheres with the corresponding 2D virtual slice (in red) for HT29 nuclear radii for a volume fraction of 0.3. (b) Corresponding projection of spheres for a $2 \mu \mathrm{m}$ slice thickness. (c) Relationship between surface and volume fractions for the simulated medium mimicking HT29 nuclei.

\section{E. Estimation of the cellular structures from the histological sections}

After the ultrasound measurements, the tissues were fixed in $10 \%$ formalin solution, processed and embedded in paraffin. Tissues were sectioned approximately in the same orientation as the ultrasound scanning planes. The slices were then stained with hematoxylin and eosin (H\&E). For each tissue (liver or mouse tumor) specimen, six histological images were selected to measure several nuclear areas using the ImageJ software. Assuming the nuclei had circular shapes, the corresponding nucleus radii were computed from the area estimates. The mean nucleus radius was estimated by measuring 3000 randomly selected cells. A procedure was then performed to obtain approximately the nucleus volume fraction from the 2D histological images, as described below. First, the number $N$ of nuclei was calculated in a given area $L_{x} \times L_{y}$ of the histological images in order to obtain the real surface fraction, as $\phi_{2 D}=N \pi a_{\mathrm{N}}^{2} /\left(L_{x} L_{y}\right)$. The calculation of the surface fractions was obtained from 15 histological images of area $150 \mu \mathrm{m} \times 150 \mu \mathrm{m}$. Secondly, a correlation between the nuclear surface and volume fractions was established for each cell category. To do this, spheres mimicking nuclei were uniformly randomly distributed within 
a simulated volume for a given volume fraction (Fig. 2(a)) using a Monte Carlo algorithm [30, section II.B.A]. The spheres had a Gaussian distribution, with the mean radius and standard deviation corresponding to those of the nuclei. Then 2D virtual slices with a thickness of $10 \mu \mathrm{m}$ (or of $2 \mu \mathrm{m}$ ) were generated from the 3D simulated volume (Fig. 2(b)). The virtual slices thicknesses are equal to those of histology: $10 \mu \mathrm{m}$ for the liver and $2 \mu \mathrm{m}$ for the mouse tumor. The surface fraction of the $2 \mathrm{D}$ virtual slices was finally calculated to be related with the volume fraction of the 3D simulated volume. This procedure was repeated several times for different volume fractions varying from 0.02 to 0.40 , and for the radii of the different categories of cells. Figure 2(c) shows an example of the relationship between surface and volume fractions for the simulated medium mimicking HT29 nuclei. It is evident that surface and volume fractions follow a linear relationship. Similar linear relationships were obtained for the radii of the different categories of cell (data not shown).

\section{RESULTS}

\section{A. Canine livers}

1) Estimation of the hepatocyte structures from the histological sections: The liver is a homogeneous tissue which consists of densely packed hepatocytes. An example of histological slice of canine liver using light microscopy is presented in Figure 3. The hepatocyte nuclei are distinctly round and the cytoplasmatic membranes are well defined. Most cells have a single nucleus, and 15 percent of them are binucleated cells [28]. On these histological slices, the nucleus and whole cells radii were estimated, along with the nucleus-to-cell-ratio. Note that this ratio for hepatocytes is quite small, around 0.35 . The volume fraction of nuclei was estimated from their surface fraction on the histological slices and the volume fraction of whole cells was calculated as $\phi_{\mathrm{C}}=0.925 \phi_{\mathrm{N}}\left(a_{\mathrm{C}} / a_{\mathrm{N}}\right)^{3}$ (where the coefficient $0.925=(1-0.15)+0.15 / 2$ corresponds to the $15 \%$ of binucleated cells). These nucleus and cells radii as well as the corresponding volume fractions are summarized in Table I.

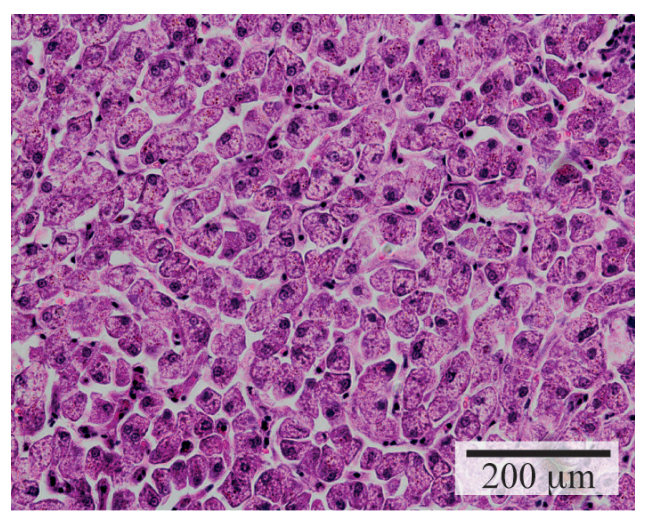

Fig. 3. Histological image of a canine liver.

2) QUS parameters: For each of the four canine livers, the QUS parameters were estimated with the SFM in the $10-38 \mathrm{MHz}$ frequency bandwidth. An example of the estimated scatterer radii $a^{*}$ and volume fraction $\phi^{*}$ 


\begin{tabular}{|c|c|c|c|c|c|}
\hline Cell & $\begin{array}{c}\text { Nucleus radius } \\
a_{\mathrm{N}}(\mu \mathrm{m})\end{array}$ & $\begin{array}{c}\text { Nucleus-to-cell } \\
\text { ratio }\end{array}$ & $\begin{array}{c}\text { Whole cell radius } \\
a_{\mathrm{C}}(\mu \mathrm{m})\end{array}$ & $\begin{array}{c}\text { Nucleus volume } \\
\text { fraction } \phi_{\mathrm{N}}\end{array}$ & $\begin{array}{c}\text { Whole cell } \\
\text { volume fraction } \phi_{\mathrm{C}}\end{array}$ \\
\hline Hepatocyte & $5.7 \pm 0.5^{*}$ & $0.35^{*}$ & $16.2 \pm 2.6^{*}$ & 0.02 & 0.43 \\
HT29 cell & $4.8 \pm 0.6^{*}$ & 0.72 & $6.7 \pm 0.8$ & 0.24 & 0.64 \\
Lymphocyte & $2.7 \pm 0.3^{*}$ & 0.67 & $4.1 \pm 0.4$ & 0.08 & 0.27 \\
\hline
\end{tabular}

TABLE 1

ESTIMATES OF THE CELLULAR STRUCTURES MEASURED OR DEDUCED FROM THE HISTOLOGICAL IMAGES FOR THE HEPATOCYTES IN THE CANINE LIVERS AND FOR THE HT29 CELLS AND NK LYMPHOCYTES IN THE MOUSE TUMORS. THE ASTERISK SYMBOL DENOTES THE VALUES ESTIMATED DIRECTLY FROM THE OPTICAL MICROSCOPE IMAGES.

estimated using "fmincon" is given in Fig. 4. This plot of data points $\left(a^{*}, \phi^{*}\right)$ displays two point clusters. The same behaviors were obtained with the four canine livers, such that the QUS parameter estimates were separated in two clusters. Therefore, data clustering was performed using the routine "fitgmdist" in MATLAB to fit Gaussian mixture distribution model with 2 components to data points $\left(a^{*}, \phi^{*}\right)$. Table II lists the QUS parameters $a^{*}, \phi^{*}$ and $\gamma_{z}^{*}$ averaged for the four canine livers and estimated with the SFM using both "fminsearch" and "fmincon". Most SFM estimates (around 65\%) were found for cluster 1 with the lowest volume fraction, whatever the optimization routine used. In the following analysis we will focus on the results obtained in cluster 1.

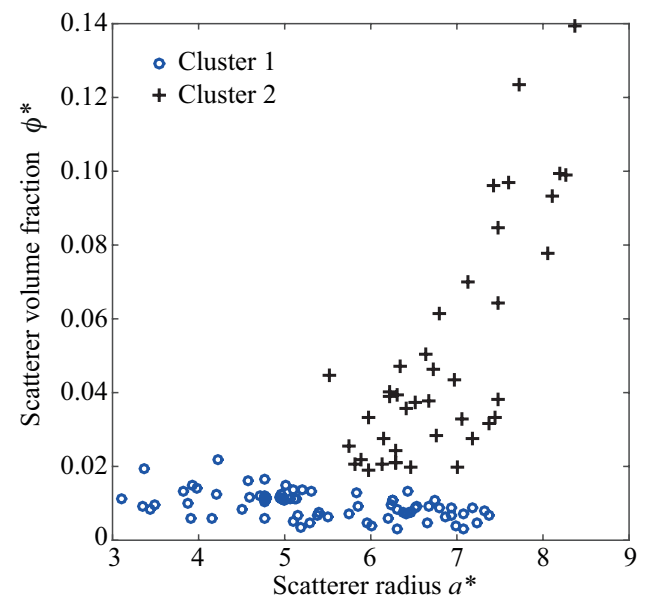

Fig. 4. Scatterer radii $a^{*}$ and volume fractions $\phi^{*}$ estimated with the SFM for liver 1 (using "fmincon"). QUS property estimates were separated in two clusters.

For the ROIs where the SFM estimates were found in cluster 1 with "fmincon", the scatterer radius $a^{*}$ and the acoustic concentration $n_{z}^{*}$ estimated from the FSM and the SGM were calculated. Figure 5 summarizes the results for the four canine livers using the three scattering models. To compare the scatterer property estimates to underlying tissue microstructures, Fig. 5 also shows in dashed lines the nuclear radius and acoustic concentration deduced from the histological analysis. The nuclear acoustic concentration deduced from histological analysis was calculated as the ratio $\phi_{N} /\left((4 / 3) \pi a_{N}^{3}\right)$ (with $a_{N}$ and $\phi_{N}$ given in Table I) multiplied by estimates for the relative impedance contrast comprised between 0.06 and 0.12 . This range of values for the relative impedance contrast 


\begin{tabular}{|c|c|c|c|c|c|c|c|}
\hline Routine & Cluster & $\begin{array}{c}a^{*} \\
(\mu \mathrm{m})\end{array}$ & $\phi^{*}$ & $\gamma_{z}^{*}$ & $\begin{array}{c}n_{z}^{*} \\
\left(\mathrm{~dB} . \mathrm{mm}^{-3}\right)\end{array}$ & $R^{2}$ & percentage \\
\hline "fminsearch" & 1 & $5.0 \pm 0.0$ & $0.0032 \pm 0.0031$ & $0.34 \pm 0.10$ & $27.27 \pm 6.33$ & $0.88 \pm 0.03$ & $63.85 \%$ \\
& 2 & $7.8 \pm 1.5$ & $0.15 \pm 0.10$ & $0.08 \pm 0.09$ & $20.70 \pm 5.68$ & $0.90 \pm 0.04$ & $36.15 \%$ \\
\hline "fmincon" & 1 & $5.3 \pm 1.0$ & $0.02 \pm 0.01$ & $0.12 \pm 0.04$ & $26.16 \pm 5.25$ & $0.89 \pm 0.04$ & $65.52 \%$ \\
& 2 & $7.4 \pm 1.4$ & $0.13 \pm 0.07$ & $0.05 \pm 0.03$ & $21.14 \pm 4.80$ & $0.89 \pm 0.04$ & $34.48 \%$ \\
\hline
\end{tabular}

MEAN eStimated VALUES OF $a^{*}, \phi^{*}, \gamma_{z}^{*}$ AND $n_{z}^{*}$ OBTAINED WITH THE SFM (USING BOTH "fminsearch" AND "fmincon") FOR THE FOUR CANINE LIVERS AND THE CORRESPONDING GOODNESS-OF-FIT $R^{2}$. THE LAST COLUMN INDICATES THE PERCENTAGE OF QUS PROPERTY ESTIMATES THAT WERE SEPARATED IN TWO CLUSTERS.

seems to be reasonable when compared with the impedance contrast of leukemia cells $\left(\gamma_{z}=0.051\right)$ [16] or of red blood cells $\left(\gamma_{z}=0.11\right)$ [31] found in the literature. The SFM, the FSM and the SGM yielded quasi-identical values of $a^{*}$ and $n_{z}^{*}$, except for liver 2 for which the acoustic concentration was slightly higher. The goodness-of-fit statistics for the three models given in Fig. 5(c) are similar, with a mean value around 0.88 .

\section{B. Mouse tumors}

1) Estimation of the cellular structures from the histological sections: Some of the mouse tumors studied here are heterogeneous because when a tumor grows, it may develop what is known as a necrotic core. An example of histological slice of such heterogeneous mouse tumor is presented on the Figure 6(a). It contains two different areas : a cellular area containing viable HT29 tumor cells and a necrotic area containing a majority of Natural Killer (NK) lymphocytes and no viable tumor cells. The nuclei boundaries of HT29 cells in the viable cellular area are well defined, but not the cytoplasmatic membranes (Fig. 6(b)). The nuclei of lymphocytes in the necrotic area are piknotic and NK lymphocytes are surrounded by fluid filled spaces that may correspond to spaces left by HT29 cells (Fig. 6(c)). The cytoplasmatic membranes were not observable for the HT29 cells and NK lymphocytes in the histological images from mouse tumors. Therefore, the nucleus-to-cell ratio of HT29 cells was estimated on a complementary experiment on fixed cells from in vitro culture, whereas the nucleus-to-cell ratio of the NK lymphocytes was deduced from the literature [29]. The volume fraction of nuclei was estimated from their surface fraction on histologic slices and the volume fraction of whole cells was calculated as $\phi_{\mathrm{C}}=\phi_{\mathrm{N}}\left(a_{\mathrm{C}} / a_{\mathrm{N}}\right)^{3}$. These nucleus and cells radii, as well as their volume fractions for HT29 cells and NK lymphocytes, are summarized in the Table I. Note that the estimations of the cellular structures could have some uncertainties since the cells/tissues shrink a little during the fixation process. In addition, the estimations of the whole NK lymphocytes may be less reliable in comparison with the other cellular structures (nuclear NK lymphocyte, or nuclear/whole HT29 cells) because the whole NK lymphocyte radius was obtained from the literature.

2) B-mode images from the mouse tumors: Experiments were conducted on growing HT29 mouse tumors. Based on the histological observations, the smaller tumors, 1, 2 and 3, were homogeneous, containing mainly viable HT29 cells while the bigger tumors, 4,5 and 6 , were heterogeneous, with the presence of several necrotic areas containing 
(a)

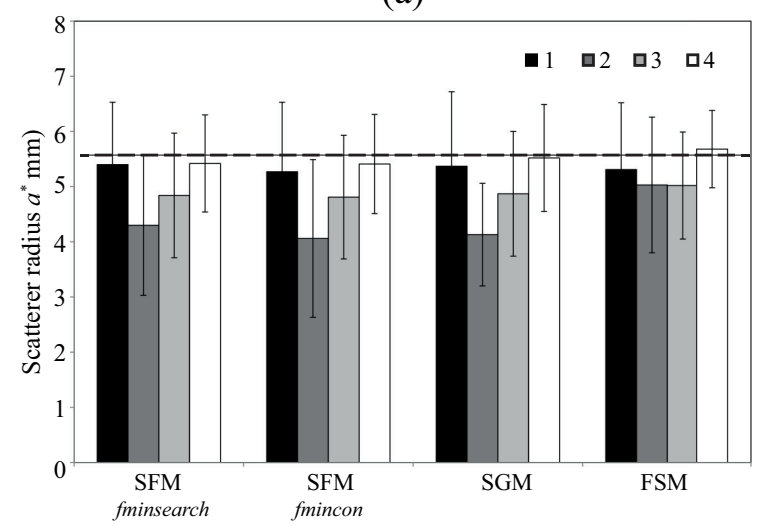

(b)

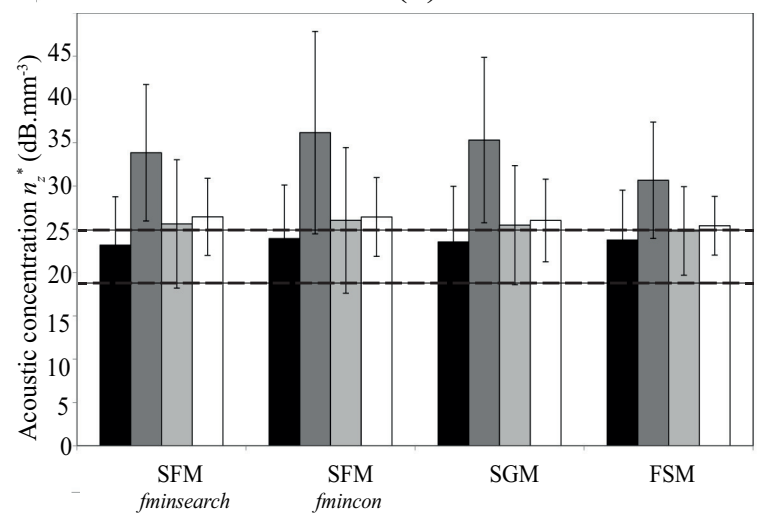

(c)

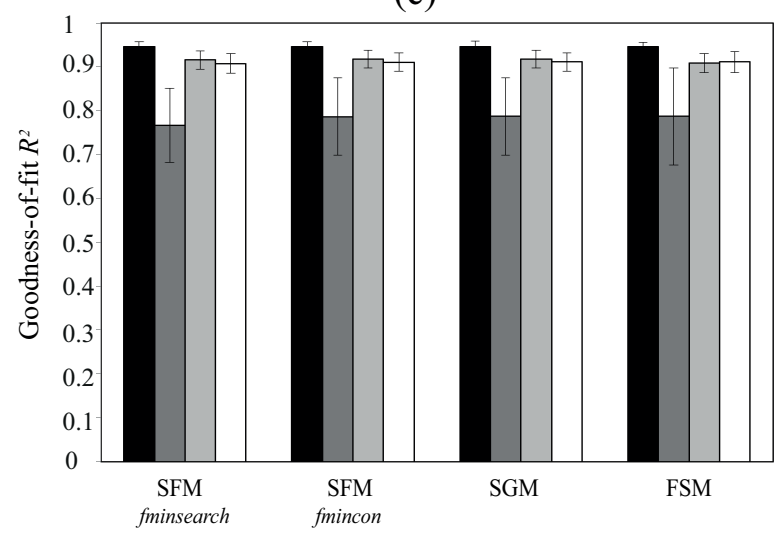

Fig. 5. (a) and (b) Structure parameters $a^{*}$ and $n_{z}^{*}$ estimated with the SFM (using both "fminsearch" and "fmincon"), the SGM and the FSM for the four canine livers. Only the SFM results found in cluster 1 are given. The dashed lines indicate the nuclear radius and acoustic concentration deduced from histological analysis. (c) Corresponding goodness-of-fit $R^{2}$.

NK lymphocytes. Figure 7 represents typical examples of histological slices from homogeneous and heterogeneous tumors, and their corresponding B-mode images. On the histological image of the heterogeneous tumor, one can observe some necrotic areas corresponding to regions no longer containing HT29 cells but infiltrated by NK cells (see also Fig. 6). The corresponding B-mode image presents a central hyperechogenic area (see Fig. 7(e)) which is 


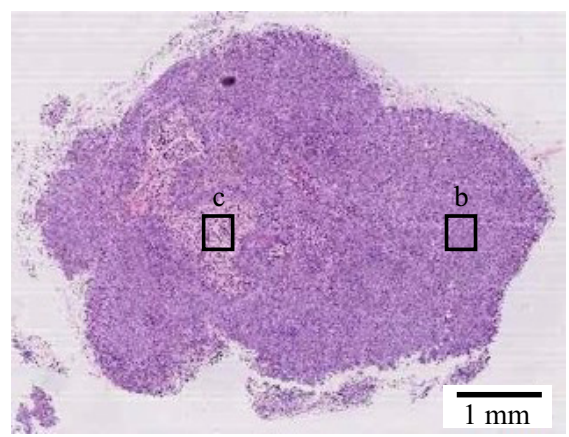

(a)

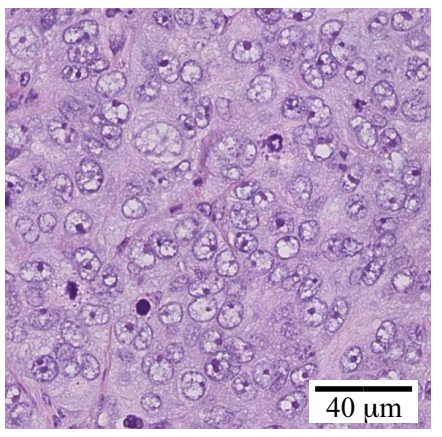

(b)

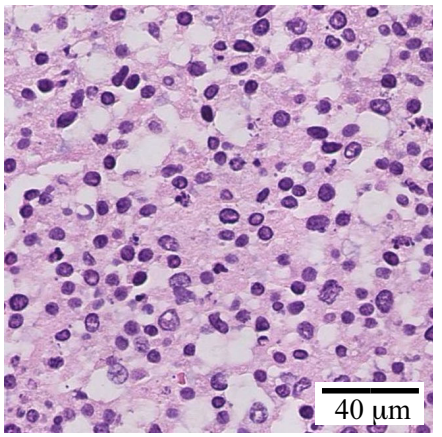

(c)

Fig. 6. (a) General view of a histological image from a heterogeneous mouse tumor containing viable and necrotic areas. Histological images of (b) a cellular area containing viable HT29 cells, and (c) a necrotic area containing a majority of NK lymphocytes.

not observed in the B-mode image of the homogeneous tumor.

(a)

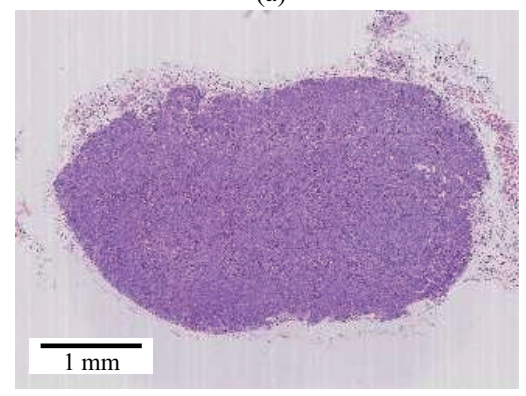

(c)

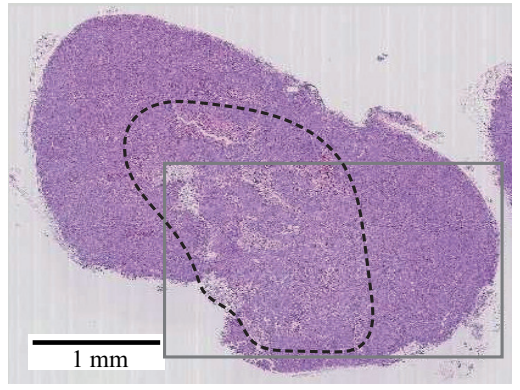

Heterogeneous tumor

Homogeneous tumor

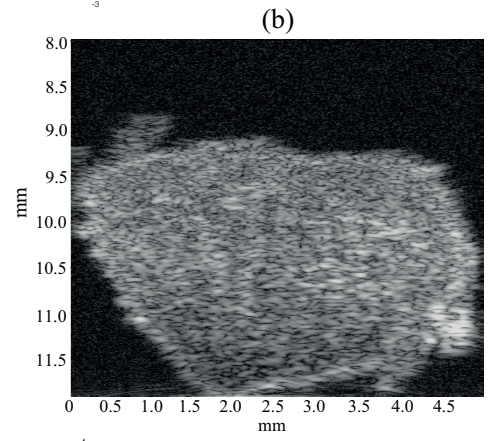

(e)

(d)

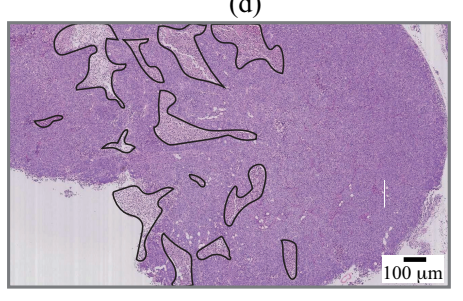

(e)

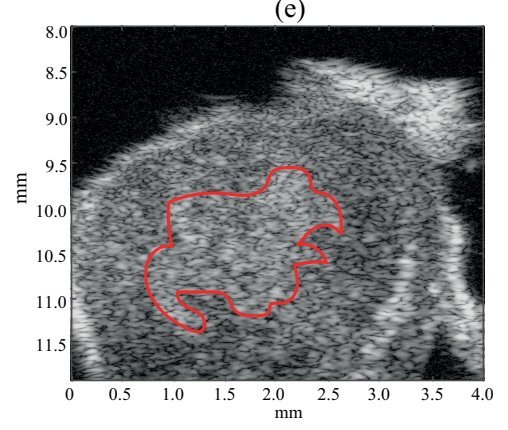

Fig. 7. (a) Histological slice from a homogeneous tumor and (b) corresponding B-mode image. (c) and (d) Histological slice from a heterogeneous tumor and (e) corresponding B-mode image. Panel (d) shows the contours of several necrotic areas and corresponds to a zoom of the histological section presented on Panel (c).

We quantified the whole tumor area as well as the necrotic area from twenty successive histological slices ( $2 \mu \mathrm{m}$ thin sections every $10 \mu \mathrm{m}$ ), as shown in Fig.7(d). For each histological slice, the ratio of the necrotic to the total 
tumor area was then calculated. A similar procedure was applied for five B-mode images to compute the ratio of the hyperechogenic to the total tumor area, as shown in Fig. 7(e). This study was performed only on tumor 4, whose central zone was sectioned into serial histological slices. The ratio obtained from the histological images was found around $0.13 \pm 0.01$, whereas the ratio estimated from the B-mode images was even greater, approximately $0.34 \pm 0.05$.

3) Spectral parameters from the homogeneous and heterogeneous tumors: The averaged ultrasound-integrated backscatter (UIB) corresponding to the averaged $B S C_{\text {meas }}$ over the analyzed frequency bandwidth (i.e., 15-42 MHz for the RMV 703) was calculated for each tumors over the entire tumor area (including the part that was necrotic in the heterogeneous tumors). The averaged UIB was found equal to $0.36 \pm 0.16,0.37 \pm 0.20$ and $0.31 \pm 0.15$ $10^{-2} \mathrm{~cm}^{-1} \cdot \mathrm{sr}^{-1}$ for tumors 1,2 and 3 , respectively, and equal to $0.79 \pm 0.41,0.90 \pm 0.56$ and $0.84 \pm 0.5210^{-2}$ $\mathrm{cm}^{-1} \cdot \mathrm{sr}^{-1}$ for tumors 4, 5 and 6 , respectively. Tumors 1, 2 and 3 present lower values of averaged UIB with a smaller variance than other tumors. Statistical significant differences were observed between the homogeneous group (tumors 1, 2 and 3) and the heterogeneous group (tumors 4, 5 and 6) when using the UIB ( $P<0.001$ with an ANOVA Kruskal-Wallis test). The enhancement of the UIB values for tumors 4, 5 and 6 is linked to the hyperechogenic areas in those tumors and corroborates the presence of necrosis, as previously observed by Sherar et al. [34].

In the following paragraphs III-B4 and III-B5, the QUS parameters of the homogeneous tumors were estimated from a gated B-mode region of the whole tumors. For the heterogeneous tumors, two gated B-mode regions were manually defined to surround a hyperechogenic area (due to the presence of some necrotic areas) and a less echogenic area that contained mainly viable HT29 cells (called in the remainder of the paper "viable HT29 cell area"). Note that both gated B-mode regions were selected as having a quite homogenous aspect.

4) QUS parameters from the viable HT29 cell areas: Figure 8 shows an example of QUS parameters estimated with the SFM using "fmincon”, as well as the UIB parameter. The scatterer radius $a^{*}$, acoustic concentration $n_{z}^{*}$ and UIB images were constructed by superimposing color-coded pixels on a conventional gray-scale B-mode image of the tumor. The size and location of the color-coded pixels corresponded to ROIs from which parameters estimates were obtained. The UIB was rather homogeneous around $0.37 \times 10^{-2} \mathrm{~cm}^{-1} \cdot \mathrm{sr}^{-1}$, the scatterers radius $a^{*}$ around $6.6 \mu \mathrm{m}$ and the acoustic concentration $n_{z}^{*}$ around $35.3 \mathrm{~dB} \cdot \mathrm{mm}^{-3}$.

When considering the SFM estimates for the mouse tumors, two clusters of data points $\left(a^{*}, \phi^{*}\right)$ were obtained (data not shown): one cluster with a high volume fraction, approximately 0.54-0.59 (called cluster 1), and another cluster with lower volume fraction, approximately 0.10 (called cluster 2), as previously observed with the canine liver experiments. Table III lists the QUS parameters $a^{*}, \phi^{*}$ and $\gamma_{z}^{*}$ averaged for the six tumors on the viable HT29 cell areas and estimated with the SFM using both "fminsearch" and "fmincon". The majority of the SFM estimates (around 87\%) was found for cluster 1, whatever the optimization routine used. Concerning the SFM estimates from cluster 1, the scatterer radius and volume fraction obtained using "fminsearch" are slightly higher than those obtained using "fmincon", but the relative impedance contrast estimates are very different using "fminsearch" and 

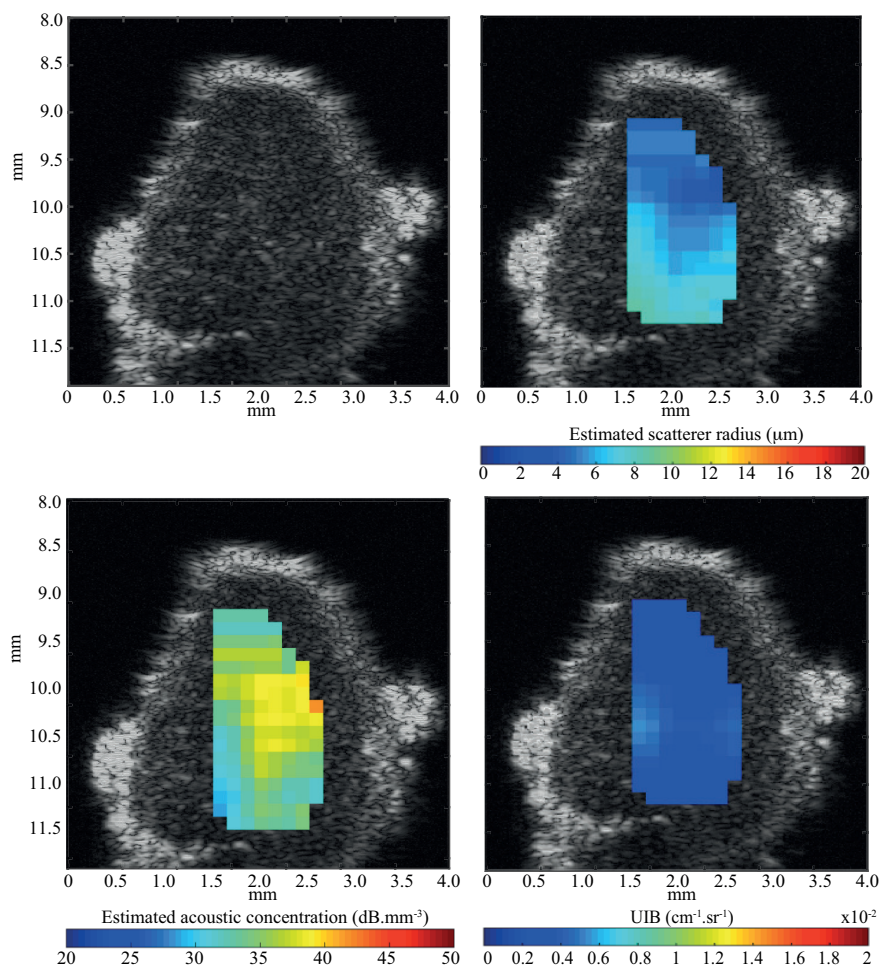

Fig. 8. Quantitative images using the SFM for a homogeneous tumor superimposed on the gray-scale B-mode image.

\begin{tabular}{|c|c|c|c|c|c|c|c|}
\hline Routine & Cluster & $\begin{array}{c}a^{*} \\
(\mu \mathrm{m})\end{array}$ & $\phi^{*}$ & $\gamma_{z}^{*}$ & $\begin{array}{c}n_{z}^{*} \\
\left(\mathrm{~dB} . \mathrm{mm}^{-3}\right)\end{array}$ & $R^{2}$ & percentage \\
\hline "fminsearch" & 1 & $8.9 \pm 2.1$ & $0.6 \pm 0.23$ & $0.43 \pm 0.88$ & $31.16 \pm 14.69$ & $0.93 \pm 0.04$ & $86 \%$ \\
& 2 & $3.6 \pm 2.0$ & $0.10 \pm 0.13$ & $0.19 \pm 0.14$ & $32.39 \pm 13.89$ & $0.91 \pm 0.04$ & $14 \%$ \\
\hline "fmincon" & 1 & $7.1 \pm 1.9$ & $0.54 \pm 0.10$ & $0.09 \pm 0.03$ & $33.82 \pm 6.55$ & $0.92 \pm 0.04$ & $87 \%$ \\
& 2 & $4.2 \pm 1.1$ & $0.10 \pm 0.07$ & $0.08 \pm 0.04$ & $30.73 \pm 6.02$ & $0.90 \pm 0.04$ & $13 \%$ \\
\hline
\end{tabular}

MeAn estimated Values of $a^{*}, \phi^{*}, \gamma_{z}^{*}, n_{z}^{*}$ OBTAINED With the SFM (USING BOth "fminsearch" AND "fmincon") FOR THE SIX TUMORS ON THE VIABLE HT29 AREAS AND THE CORRESPONDING GOODNESS-OF-FIT $R^{2}$. THE LAST COLUMN INDICATES THE PERCENTAGE OF QUS PROPERTY ESTIMATES THAT WERE SEPARATED IN TWO CLUSTERS.

"fmincon". Table IV also lists the QUS parameters $a^{*}, \phi^{*}, \gamma_{z}^{*}, n_{z}^{*}$ and UIB estimated with the SFM from cluster 1 (using "fmincon") for each mouse tumor in the viable HT29 cell areas.

For the ROIs where the SFM estimates were found in cluster 1 with "fmincon", the QUS parameters from the SGM and the FSM are estimated. The scatterer radius $a^{*}$ and the acoustic concentration $n_{z}^{*}$ for the SFM, the SGM and the FSM are presented in Fig. 9. Also given in Fig. 9 in dashed lines are the whole cell radius and acoustic concentration deduced from the histological analysis in order to compare the QUS parameter estimates to the underlying tissue microstructures. The QUS parameters estimated with the sparse SGM and FSM are very different from the concentrated SFM. The comparison between the goodness-of-fit statistics for the three models 
given in Fig. 9 shows that the concentrated SFM had the best fit to the experimental data $\left(R^{2} \approx 0.93\right)$ followed by the sparse $\operatorname{SGM}\left(R^{2} \approx 0.86\right)$.

(a)

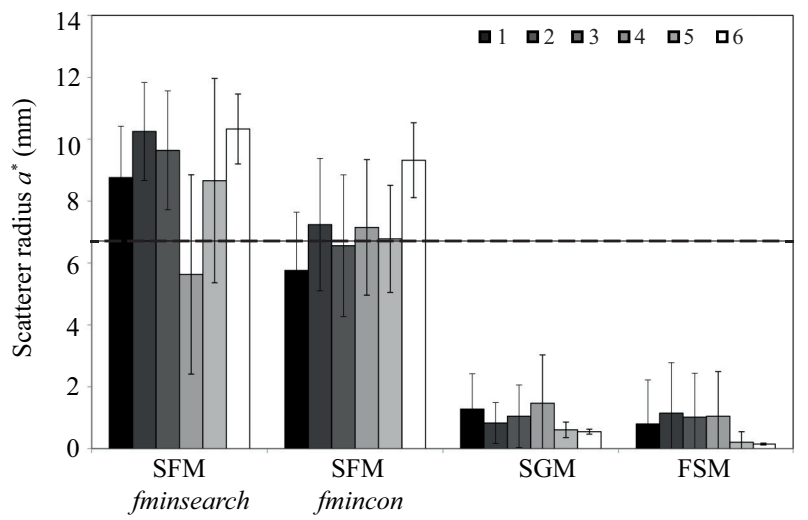

(b)

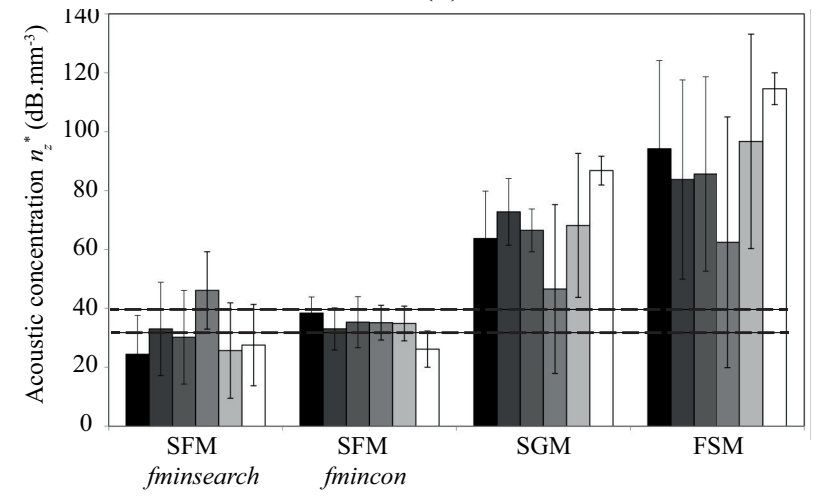

(c)

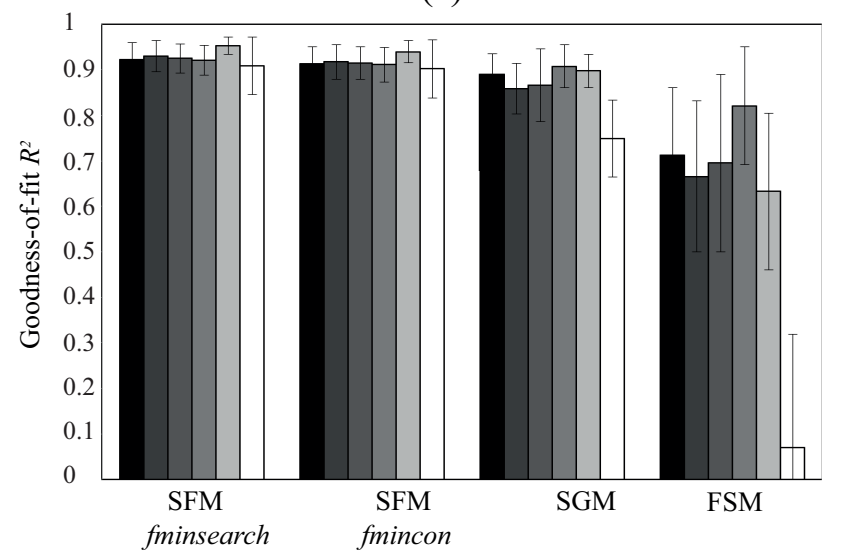

Fig. 9. (a) and (b) Structure parameters $a^{*}$ and $n_{z}^{*}$ estimated with the SFM (using both "fminsearch" and "fmincon"), the SGM and the FSM on the viable HT29 cell areas for the six tumors. Only the SFM results found in cluster 1 are given. The dashed lines indicate the whole cell radius and acoustic concentration deduced from histological analysis. (c) Corresponding goodness-of-fit $R^{2}$. 
5) QUS parameters from the hyperechogenic areas: An example of QUS parameter images obtained with the SFM is given in Figure 10 for a heterogeneous tumor composed of a hyperechogenic area (due to the presence of some necrotic areas) and a less echogenic area (containing mainly viable HT29 cells). Figure 10 reveals that the scatterer radii estimated with the SFM were quite similar in both areas, but the acoustic concentrations were slightly higher in the less echogenic area when compared with the hyperechogenic area. The UIB is higher in the hyperechogenic area $\left(>1.1 \times 10^{-2} \mathrm{~cm}^{-1} \cdot \mathrm{sr}^{-1}\right)$ than in the viable HT29 cell area $\left(<0.8 \times 10^{-2} \mathrm{~cm}^{-1} \cdot \mathrm{sr}^{-1}\right)$.
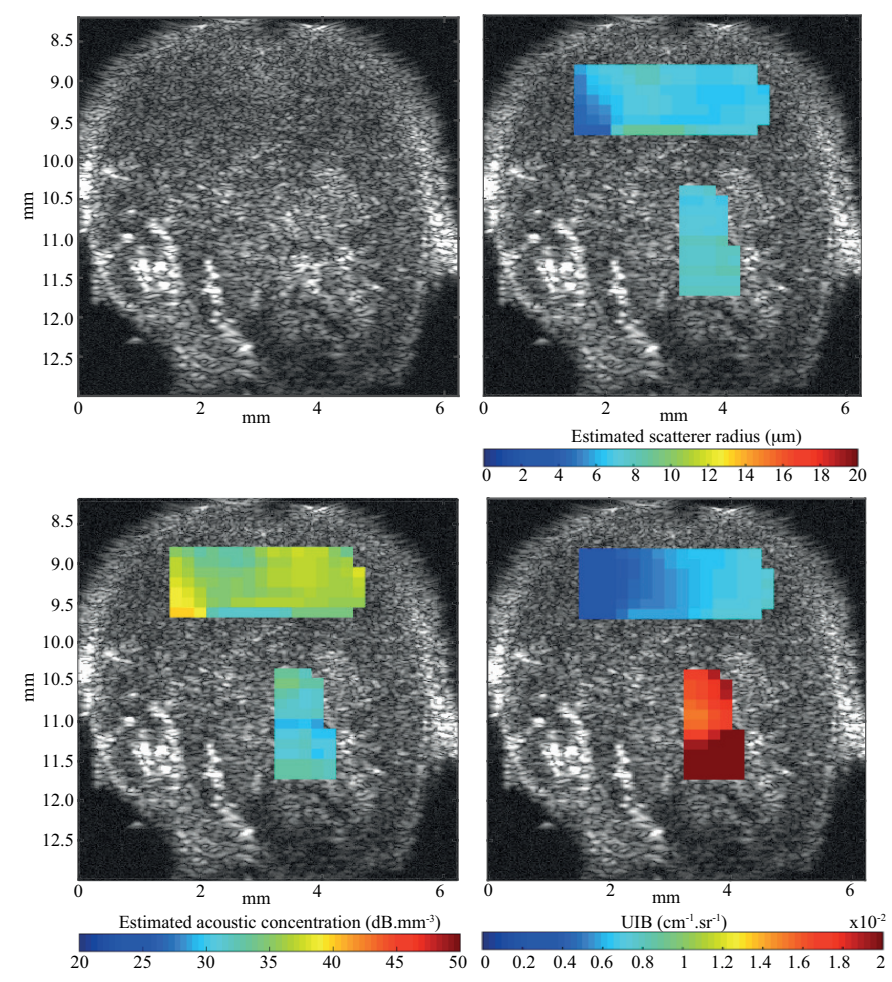

Fig. 10. Quantitative images using the SFM for a heterogeneous tumor superimposed on the gray-scale B-mode image.

The QUS parameters estimated with the SFM from cluster 1 (using "fmincon") are given in Table IV for each mouse. For the ROIs where the SFM estimates were found in cluster 1 with "fmincon", the QUS parameters from the SGM and the FSM are estimated. The scatterer radius $a^{*}$ and the acoustic concentration $n_{z}^{*}$ estimated by the SFM, the SGM and the FSM are presented in Fig. 11. The goodness-of-fit statistics reveals that the concentrated SFM gave satisfactory fitting curves $\left(R^{2} \approx 0.90\right)$ followed by the sparse SGM $\left(R^{2} \approx 0.84\right)$.

\section{Discussion}

\section{A. Canine livers}

1) Comparison between sparse and concentrated models: The scatterer radius $a^{*}$ and the acoustic concentration $n_{z}^{*}$ obtained from the FSM, SGM and SFM are quasi-identical, as shown in Fig. 5. This had been observed on 


\begin{tabular}{|c|c|c|c|c|c|c|c|}
\hline Tumor & Area & $\begin{array}{c}a^{*} \\
(\mu \mathrm{m})\end{array}$ & $\phi^{*}$ & $\gamma_{z}^{*}$ & $\begin{array}{c}n_{z}^{*} \\
\left(\mathrm{~dB} . \mathrm{mm}^{-3}\right)\end{array}$ & $\begin{array}{c}R^{2} \\
\left(10^{-2} \mathrm{~cm}^{-1} . \mathrm{sr}^{-1}\right)\end{array}$ \\
\hline 1 & Viable HT29 cell area & $5.8 \pm 1.9$ & $0.52 \pm 0.10$ & $0.10 \pm 0.03$ & $38.40 \pm 5.48$ & $0.40 \pm 0.18$ & $0.91 \pm 0.04$ \\
2 & Viable HT29 cell area & $7.2 \pm 2.1$ & $0.55 \pm 0.12$ & $0.08 \pm 0.03$ & $33.05 \pm 7.19$ & $0.43 \pm 0.23$ \\
3 & Viable HT29 cell area & $6.6 \pm 2.3$ & $0.55 \pm 0.11$ & $0.09 \pm 0.04$ & $35.32 \pm 8.68$ & $0.37 \pm 0.16$ \\
4 & Viable HT29 cell area & $7.2 \pm 2.2$ & $0.57 \pm 0.09$ & $0.09 \pm 0.02$ & $35.14 \pm 5.89$ & $0.50 \pm 0.09$ \\
5 & Viable HT29 cell area & $6.8 \pm 1.7$ & $0.53 \pm 0.10$ & $0.09 \pm 0.03$ & $34.87 \pm 5.90$ & $0.61 \pm 0.32$ \\
6 & Viable HT29 cell area & $9.3 \pm 1.2$ & $0.53 \pm 0.08$ & $0.08 \pm 0.03$ & $26.16 \pm 6.16$ & $1.40 \pm 0.62$ \\
\hline 4 & Hyperechogenic area & $7.0 \pm 1.6$ & $0.50 \pm 0.12$ & $0.10 \pm 0.03$ & $35.32 \pm 4.71$ & $1.00 \pm 0.34$ \\
5 & Hyperechogenic area & $7.7 \pm 1.2$ & $0.49 \pm 0.10$ & $0.09 \pm 0.02$ & $32.76 \pm 4.30$ & $1.54 \pm 0.52$ \\
6 & Hyperechogenic area & $7.9 \pm 2.0$ & $0.48 \pm 0.12$ & $0.09 \pm 0.03$ & $31.81 \pm 6.59$ & $0.93 \pm 0.09$ \\
\hline
\end{tabular}

Estimated VALUES OF $a^{*}, \phi^{*}$ AND $\gamma_{z}^{*}$ AND UIB OBTAINED WITH THE SFM FROM CLUSTER 1 (USING ”fmincon") FOR THE VIABLE HT29 CELL AREAS AND THE HYPERECHOGENIC AREAS AND THE CORRESPONDING GOODNESS-OF-FIT $R^{2}$.

diluted cell pellet biophantoms experiments for cell volume fractions less than 0.06 [17, Fig. 7]. Indeed, when the medium is diluted, the structure factor is close to the unity value, and the concentrated SFM is equivalent to the sparse FSM modeling. In addition, the goodness-of-fit statistic reveals that all the models fit well the measured data. Thus, the similarities of the QUS estimates and of the goodness-of-fit statistics obtained with the sparse and concentrated models suggest that the liver is a diluted medium (with low scatterer volume fraction).

Moreover, the sparse models (SGM and FSM) and the SFM from cluster 1 give a mean scatterer radius around $5.3 \mu \mathrm{m}$ that closely matches the true radius of hepathocyte nuclei with a difference around $8 \%$. The mean acoustic concentration (around $26 \mathrm{~dB} . \mathrm{mm}^{-3}$ ) estimated with the three models agrees well with the nuclear acoustic concentration of $25 \mathrm{~dB} \cdot \mathrm{mm}^{-3}$ deduced from histological analysis. These findings also corroborate our suggestion that the liver is a diluted medium. Indeed, if the nuclei (and not the whole cells) are the cellular structures responsible for scattering, the corresponding scatterer volume fraction is expected to be approximately 0.02 (see Table I). To conclude, the QUS parameter estimates from the three scattering models suggest that the nucleus is the dominant source of scattering in the liver in this frequency range. This conclusion was suggested previously in a numerical study based on 3D impedance maps of rabbit liver [32].

2) Simultaneous estimation of the volume fraction and relative impedance contrast using the SFM: One of the characteristics of the SFM is its ability to provide the volume fraction and the relative impedance contrast (whereas the sparse models SGM and FSM provide only the acoustic concentration). The frequency dependent BSC behavior depends on the product $\sigma_{b}(k, a) S(k, a, \phi)$, whereas it depends only on $\sigma_{b}(k, a)$ when using the sparse models (SGM or FSM). This ability was studied here on the canine liver data, as shown in Table II.

For the SFM estimates from cluster 1, both optimization routines gave similar scatterer radii and acoustic concentrations. Moreover, the mean scatterer radius agrees well with the true nuclear radius of hepathocyte. However, the volume fraction and impedance contrast are very different depending upon the optimization routine. When using "fminsearch", the mean volume fraction was six times smaller than the nuclear volume fraction estimated from 
(a)

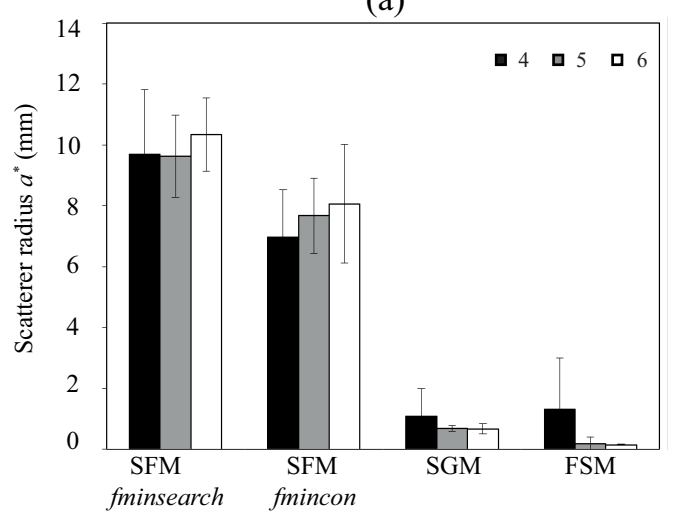

(b)

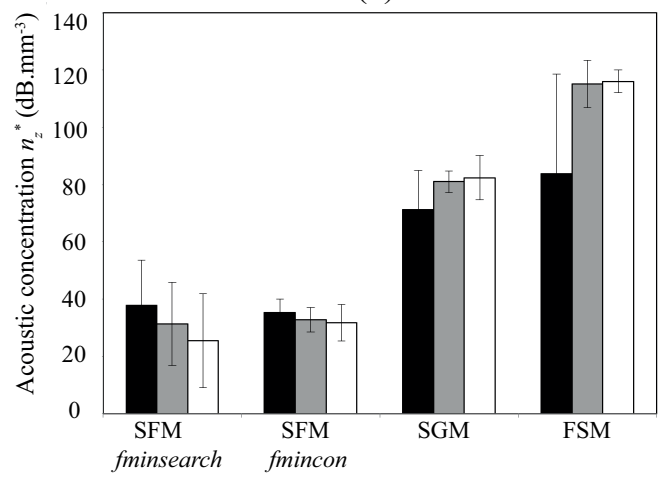

(c)

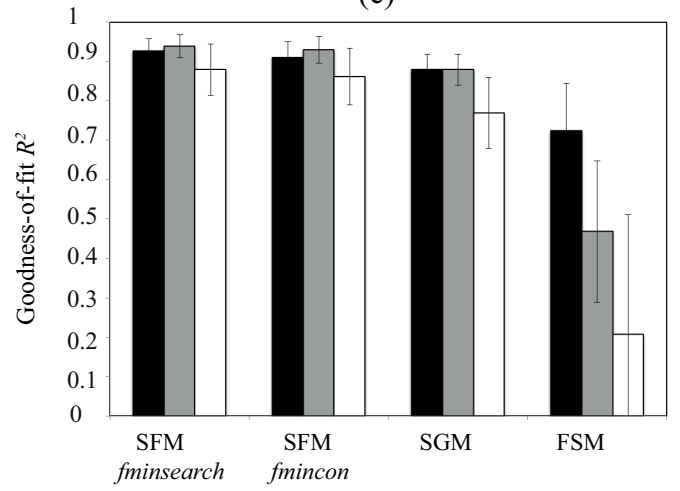

Fig. 11. (a) and (b) Structure parameters $a^{*}$ and $n_{z}^{*}$ estimated with the SFM (using both "fminsearch" and "fmincon"), the SGM and the FSM on the hyperechogenic areas for the three heterogeneous tumors. (c) Corresponding goodness-of-fit $R^{2}$.

histology and the impedance contrast was higher, around 0.34. Note that we empirically found that "fminsearch" provides the global minimum, as observed by plotting some cost function surfaces. An example is given in Fig. 12. As expected, several couples ( $\phi$ and $\gamma_{z}$ ) gave the same BSC behavior and quasi-identical cost function values, as shown in Figs. 12(a) and 12(c). When using "fmincon", excellent agreements were obtained between the nuclear structures (radius and volume fraction) estimated by ultrasound and by histology. The mean volume fraction was found equal to 0.024 and agrees well with the nuclear volume fraction of approximately 0.02 estimated from 
histological analysis. The mean impedance contrast was found equal to 0.12 and seems to be in a reasonable range of values [16] [31].
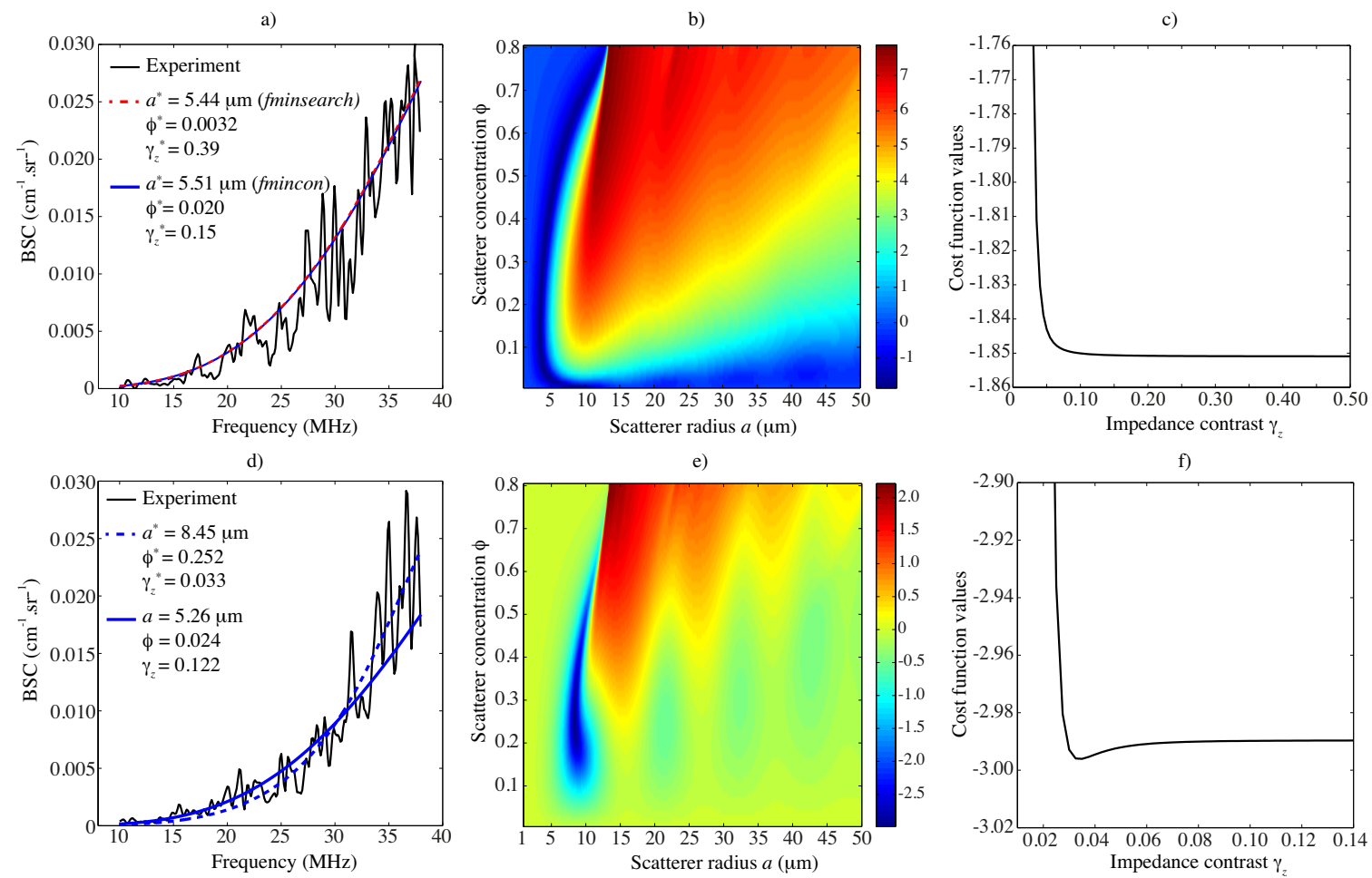

Fig. 12. (a) Example of measured BSC and corresponding fitted curve obtained with the SFM using both "fminsearch" and "fmincon". (b) Logarithm of the cost function $F(a, \phi)$ for a fixed value of $\gamma_{z}^{*}=0.15$ for the SFM. The logarithm is shown here in order to increase the visual contrast. (c) Cost function $F\left(a^{*}, \phi^{*}, \gamma_{z}\right)$ with the SFM for varying values of $\gamma_{z}$ ( $a^{*}$ and $\phi^{*}$ being calculated for each fixed value of $\gamma_{z}$ ). (d) Example of measured BSC for cluster 2 and corresponding fitted curve obtained with the SFM (dashed line). Also represented in solid line is the theoretical $B S C_{\mathrm{SFM}}$ curve calculated with the mean QUS parameters of the SFM for cluster 1. (e) Same as (b) for $\gamma_{z}^{*}=0.033$. (f) Same as (c).

The SFM estimates from cluster 2 are quasi-identical whatever the optimization routine tested (i.e., "fminsearch" or "fmincon"). The mean scatterer radius estimate was comprised between 7.4 and $7.8 \mu \mathrm{m}$, which is slightly larger than the actual nuclear radii of approximately $5.7 \mu \mathrm{m}$. The mean volume fraction estimate was six times larger than the nuclei volume fraction and three times smaller than the whole cell volume fraction. Thus, the estimates from cluster 2 did not match any microstructural characteristics observed from histological images. There could be several meanings for the estimates from cluster 2. They could be attributed to the presence of other possible scattering sources such as nucleus aggregates of binucleated cells or microvessels. The difficulty in simultaneously evaluating three parameters that can have the same effect on the BSC might as well contribute to the estimates from cluster 2, as explained below. Figure 13 illustrates the respective effect of varying $a, \phi$ and $\gamma_{z}$ on the BSC, a $\log -\log$ scale was chosen to better visualize the BSC frequency dependence and the spectral slope. An increase in $a$ has the effect of decreasing the spectral slope and increasing the amplitude, whereas an increase in $\phi$ has the effect 
of increasing the spectral slope. A decrease in $\gamma_{z}$ has the effect of decreasing the BSC amplitude at all frequencies. That is why the overestimation of $\phi$ goes with the overestimation of $a$, and the overestimation of $a$ goes with an underestimation of $\gamma_{z}$ for the estimates of cluster 2 (when compared with cluster 1).

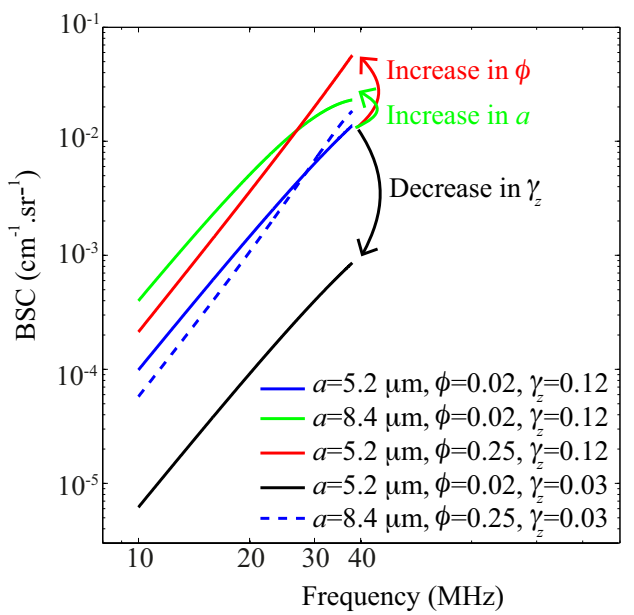

Fig. 13. Effect of increasing $a$ and $\phi$ and of decreasing $\gamma_{z}$ on the BSC, when using the SFM.

Finally, one might think that the estimates from cluster 2 correspond to cost functions having several local minima. Figure 12(d) represents an example of measured $B S C_{\text {meas }}$ curve and the corresponding fitted curve obtained from the SFM (dashed line). The estimates belong to the QUS data from cluster 2. The corresponding cost function of the fitted data from cluster 2 displayed in Fig. 12(e) exhibits several local minima. The global minimum was satisfactorily estimated with the minimization procedure. However, neither the global minimum nor the local minimum is correlated with the true nuclear structures. Also represented in solid line in Fig. 12(d) is the theoretical $B S C_{\mathrm{SFM}}$ curve calculated with the mean QUS parameters obtained with cluster 1 using ”fmincon” (see Table II). Although both theoretical curves are quite similar, the QUS parameters $a^{*}, \phi^{*}$ and $\gamma_{z}^{*}$ from clusters 1 and 2 are different.

\section{B. Mouse tumors}

1) QUS parameters from the viable HT29 cell areas: For the viable HT29 areas, the goodness-of-fit statistic reveals that the SFM had the best fit to the experimental data (Fig. 9). In addition, large differences in the QUS parameter estimates were obtained depending upon whether a sparse or concentrated model is used. The mean scatterer radius obtained with the sparse SGM and FSM are equal to 1.0 and $0.7 \mu \mathrm{m}$, respectively, and are not correlated with the cellular structures. In our previous experiments on cell pellet biophantoms, it was observed that the sparse models underestimated scatterer radius when the volume fraction was greater than 0.12 [17, Fig. 7]. The same behavior was obtained in the present study on ex vivo mouse tumors. The goodness-of-fit parameters obtained 
with the concentrated SFM and the differences between sparse and concentrated models suggest that the viable HT29 areas could be considered as concentrated media with densely packed cells.

The SFM estimates from cluster 1 represent the majority (around 86\%) of the estimates. The mean scatterer radius estimated with the SFM was found equal to $7.1 \mu \mathrm{m}$ using "fmincon" and $8.9 \mu \mathrm{m}$ using "fminsearch", which is slightly larger than the whole HT29 cell radius, with relative errors of 7\% and 32\%, respectively. The scatterer volume fraction matches quite well the whole cell volume fraction deduced from histological analysis, with relative errors less than $15 \%$, whatever the optimization routine tested. Using "fminsearch", the impedance contrast is overestimated, when compared with the cellular impedance contrast values comprised between 0.05 and 0.11 found in the literature [16], [31]. Overall, excellent agreements were obtained between the cellular structures estimated by ultrasound and the whole cellular structures deduced from histological observations using the SFM with "fmincon". The scatterer radius and volume fraction estimated with the SFM using "fminsearch" are also quite satisfactory. This finding suggests the whole HT29 cell is the dominant scattering source in the HT29 mouse tumors.

For both "fminsearch" and "fmincon" routines, the SFM estimates from cluster 2 gave a mean scatterer radius comprised between 3.6 and $4.2 \mu \mathrm{m}$, which is slightly smaller than the HT29 nuclear radius. However, the mean scatterer volume fraction was approximatively 0.10 , which does not match the HT29 nuclear volume fraction with relative error of $58 \%$. So the estimates from cluster 2 did not represent the actual microstructural parameters of viable HT29 cells, whatever the optimization routine tested.

2) B-mode images and QUS parameters from the hyperechogenic areas: The ratios between the necrotic and whole tumor areas obtained from the B-mode images (0.34) and from the histological images (0.13) were very different. This discrepancy could be due to small necrotic areas close to each other. If the distance between necrotic areas is smaller than the lateral resolution of the RMV703 probe (equal to $110 \mu \mathrm{m}$ ), these areas cannot be discriminated on the B-mode images. In addition, the spaces between necrotic areas could contain HT29 cells undergoing a cell death process that could also contribute to the increase in echogenicity [12]. To test these hypotheses, we delimited a larger area that surrounds all the small necrotic areas, as shown in Fig. 7(c). The new ratio was found equal to $0.32 \pm 0.05$, which is close to the hyperechogenic area value found on the B-mode images. The large hyperechogenic area found in the B-mode image may be composed of a mix of HT29 cells (viable or undergoing a cell death process) and NK lymphocytes. The difference between the new ratio (0.32) and the initial estimated ratio (0.13) from the histological images suggests that both cell lines occupy an equivalent proportion in the large hyperechogenic area.

When estimating the QUS parameters from the large hyperechogenic area, the scatterer radius and acoustic concentration from the necrotic area differed depending upon whether a sparse or concentrated model is used (see Fig. 11). So this tissue could be considered as a concentrated medium. The QUS parameters estimated with the SFM showed slightly larger scatterer radius and smaller volume fraction when compared with the QUS parameters from the viable HT29 areas (see Table IV). One can notice that the radius and volume fraction estimated from the 
hyperechogenic areas are closer to the true structures of whole HT29 cells than to the structures of lymphocytes, since the lymphocytes are smaller than the HT29 cells. Based on the SFM estimates, one could think that the HT29 is the dominant scattering cell line, but it is not consistent with the differences observed on the UIB values between the hyperechogenic areas and the viable HT29 areas. It is difficult to compare the QUS estimates obtained from the SFM and the cellular structures observed in histological slices in the hyperechogenic area. First, this difficulty could be explained by the complex histopathological features: the large ROI sizes (at least $600 \mu \mathrm{m} \times 500 \mu \mathrm{m}$ ) used for the ultrasonic data analysis contain small necrotic areas surrounded by HT29 cell areas (see Fig. 7(d)). Based on the histological observations, several components could be responsible for scattering: the viable HT29 cell, the HT29 cells undergoing a cell death process, the NK lymphocytes (in the necrotic areas) and the dispersed cytoplasmic fluid (in the necrotic areas). In addition, not all changes observed on optical histology will result in scattering changes. Second, the SFM used in the present study considers identical scatterers and could fail to estimate scattering structures from mixtures of scatterers differing in size and/or in acoustic properties. The understanding of the QUS parameters estimated with the SFM and their relationship with histological features are thus difficult. We try herein to explain the increase in UIB observed in the hyperechogenic areas within the framework of a discrete-scattering approach. As a first approximation, the tissue was modeled as a two-component scattering medium. The two types of scatterers involved were the whole HT29 cells and the (nuclear or whole) NK lymphocytes, since the nuclear or whole cells are generally assumed to be the dominant source of scattering in the discrete-scattering approach [4], [12], [16]. Figure 14 compares the predicted BSC (using the SFM) from the nuclear and whole NK lymphocytes, and from the whole HT29 cells. The BSC curves were computed for two extreme values of relative impedance contrast (0.06 and 0.12). It is interesting to observe that for the same relative impedance contrast, both nuclear lymphocytes and whole HT29 cells give similar BSC behaviors (based on the SFM theory). Small nuclear structures (of $2.7 \mu$ m radius) with low volume fraction could have the same BSC magnitude as densely packed whole HT29 cells (of $6.7 \mu \mathrm{m}$ radius). Based on the BSC amplitudes obtained from several types of scatterers given in Fig. 14, the UIB differences between the hyperechogenic areas and the viable HT29 areas could be explained by the difference between BSC curves, considering:

(1) either the whole lymphocytes $\left(a=4.1 \mu \mathrm{m}, \phi=0.24, \gamma_{z}=0.06\right.$ or 0.12$)$ and the whole HT29 cells $(a=6.7 \mu \mathrm{m}$, $\phi=0.64, \gamma_{z}=0.06$ or 0.12 ),

(2) or the nuclear lymphocytes with large impedance contrast ( $\left.a=2.7 \mu \mathrm{m}, \phi=0.08, \gamma_{z}=0.12\right)$ and the whole HT29 cells with low impedance contrast $\left(a=6.7 \mu \mathrm{m}, \phi=0.64, \gamma_{z}=0.06\right)$.

Future work should be conducted to go further into the understanding of the scattering from necrotic areas. For example, based on our hypothesis of a tissue being modeled as a two-component scattering medium, the SFM should be developed to take into account two populations of scatterers differing in size (and/or in acoustic properties) using a bidisperse structure factor [33].

Sherar [34] has studied the ultrasound backscatter from human bladder cell (MGH-U1) tumor spheroid and observed a higher backscatter from necrotic areas. In this study, the higher backscatter was interpreted as large acoustic impedance variations between collapsed nuclear chromatin and the surrounded liquid matrix, as the necrotic 
regions are composed of pyknotic nuclei surrounded by large spaces containing dispersed cytoplasmic fluid. The explanation provided by Sherar [34] is close to our second hypothesis, except that our approach also includes the scatterer radius and volume fraction which are of major interest when dealing with densely packed tumor cells.

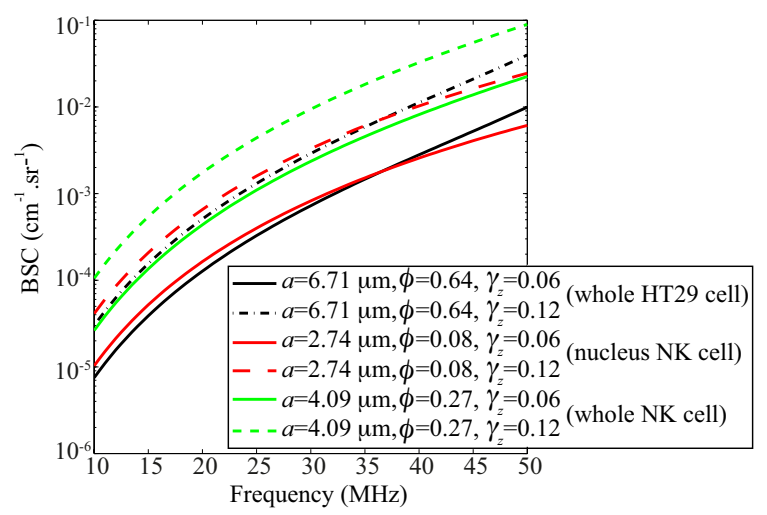

Fig. 14. Predicted theoretical BSC using the SFM from the nuclear and whole NK lymphocytes as well as the whole HT29 cells.

\section{On the choice of the "fminsearch" and "fmincon" routines}

Two routines ("fminsearch" and "fmincon") were studied to obtain the QUS parameters with SFM. As shown in Figure 5, 9 and 11, both routines gave similar $R^{2}$ values, whatever the tissue studied. The scatterer radius and acoustic concentration estimated with both routines were also within the same range of values for each cluster respectively (see also Tables II and III). However, the volume fraction and the relative impedance contrast differ depending on the routine used. Investigators interested in using the SFM for clinical studies have to decide which routine to use. On one side, the "fminsearch" routine empirically provided the global minimum (as observed by plotting the cost function surfaces - Figure 6), but could result in unrealistic values with the underestimation of the volume fraction ( $\phi=0.0032$ for the liver - Table II) or the overestimation of the impedance contrast $\left(\gamma_{z}=0.43 \pm 0.88\right.$ for the mouse tumors - Table III). On the other side, a major constraint when using the "fmincon" routine was put on the relative impedance contrast comprised between 0 and 0.2 , but the QUS parameters estimated from cluster 1 matched quite well the cellular structures obtained from histology. (Note that the other conditions $0 \leq a \leq 100 \mu \mathrm{m}$, $0 \leq \phi \leq 1$ did not greatly constrain the minimization routine.) Therefore both routines could be used to determine the scatterer radius and acoustic concentration, but the "fmincon" routine should probably be preferred to estimate the QUS parameters $\left(a^{*}, \phi^{*}\right.$ and $\left.\gamma_{z}^{*}\right)$. For in vivo clinical applications, the estimates from cluster 2 could bias the QUS parameters but the present study shows that these estimates represent only 35\% and $14 \%$ for the liver and the mouse tumor, respectively. As discussed previously in section IV-A2, there are several meanings for the estimates from cluster 2: the presence of other possible scattering sources (microvessels or cell aggregates, etc) or the difficulty to estimate three parameters simultaneously. 


\section{On the use of the structure factor model}

The comparison of the goodness-of-fit statistics between the sparse and concentrated models shows the superiority of the concentrated SFM to explain the measured BSC in densely-packed media. The values of $R^{2}$ obtained with the SFM are around 0.89 for the livers, 0.93 for the viable HT29 areas and 0.90 for the hyperechogenic areas. It is interesting to observe that the sparse and concentrated models gave similar $R^{2}$ statistics and identical QUS parameters (scatterer radius and acoustic concentrations) for the canine liver experiments. Since both sparse and concentrated models explained the measured BSC very well, this tissue could be considered as a diluted medium. On the contrary, the sparse and concentrated models yield different $R^{2}$ statistics and QUS parameters for the homogeneous mouse tumors, and the SFM provides the best fit to the data. This suggests that the SFM is the most adequate to estimate the QUS parameters for cellular structures and that the HT29 mouse tumors could be considered as a concentrated medium.

For the canine liver, the SFM estimates matched well the nuclear structures obtained from histological analysis (with relative errors less than $7 \%$ on the scatterer radius and volume fraction). This is corroborated by the similar QUS parameters estimated with the three scattering models meaning that the medium may be diluted. These results demonstrate that the nucleus could be a dominant source of scattering in liver. For the viable HT29 cell areas, scatterer radius and volume fraction estimated with the SFM showed good agreement (with relative errors less than 15\%) with the HT29 cellular structure obtained from histological analysis, whereas the sparse models (SGM and FSM) gave no consistent QUS parameters. This suggests that the viable HT29 cell areas have densely packed cellular content and that the whole HT29 cell is responsible for the scattering. Concerning the hyperechogenic areas (containing several small necrotic areas with NK lymphocytes surrounded by HT29 cell areas), QUS parameter estimates with the SFM were difficult to interpret because of the presence of two cell types (HT29 cells and NK lymphocytes). However, as the sparse models provide smaller scatterer radius values and higher acoustic concentration values as for HT29 viable cells areas, these hyperechogenic areas could be considered as a dense medium. Future studies should focus on the understanding of the necrotic process by conducting controlled experiments on cell pellet biophantoms and on the improvement of the SFM to take into account the tumor heterogeneity of the cell types.

High ultrasonic frequencies are required to estimate microstructural parameters at the level of the nuclei or cells. It is well known that the most accurate measurements are obtained in the range of $0.5<k a \leq 1.2$ (with $k a$ the product of the wavenumber and the scatterer radius) [35]. In this range, the size of the scatterer is the principal factor determining the frequency dependence of the BSC when using the sparse models. At the center frequency of $25 \mathrm{MHz}$ in livers (or at the center frequency of $30 \mathrm{MHz}$ in mouse tumors), QUS is thus most sensitive to scatterer radius comprised between 4.8 and $11.5 \mu m$ (or between 4.0 and $9.5 \mu m$, respectively). Insana and Hall [35] have reported that for $k a<0.5$ (the Rayleigh scattering regime), the noise of the spectral estimate is much greater that the changes due to the scatterer structure and measurement sensitivity is lost. In the present study, the scatterer radius estimates less than $4.8 \mu \mathrm{m}$ in livers or less than $4.0 \mu \mathrm{m}$ in mouse tumors (corresponding to $k a<0.5$ ) are likely erroneous. It is important to notice that when using the SFM for examining densely-packed media, both 
scatterer radius and volume fraction could strongly modify the frequency dependence of the BSC (that depends on the fluid sphere form factor $F F(k, a)$ and the structure factor $S(k, a, \phi))$. Franceschini et al. recently demonstrated the SFM superiority over sparse scattering models (such as the SGM or FSM) to estimate the radius and acoustic concentration for a product $k a$ around 0.7 , especially for concentrated media with volume fractions $\phi \geq 0.12$ [17]. Further studies are required to determine the accuracy of QUS parameters estimated using the SFM for wider $k a$ range and for several scatterer size distributions and volume fractions.

\section{Conclusion}

In summary, for both canine livers and viable HT29 cell areas, the QUS parameters estimated with the SFM were found to be well correlated to the true nuclear or cellular structures, and the SFM (using "fmincon") was able to provide quantitatively satisfactory estimates for the volume fraction and the relative contrast impedance (instead of only the acoustic concentration for the sparse models). This study demonstrated that the SFM is a moreappropriate model to use for modeling densely packed cellular content in HT29 mouse tumors. The concentrated SFM provides better fit when compared with classical models (SGM, FSM) valid for diluted media. The SFM model makes it possible to estimate structure parameters close to cellular structures on the homogeneous HT29 mouse tumors, which the classical sparse models (SGM, FSM) are incapable of. The SFM has been initially developed for characterizing red blood cell aggregation in flowing blood [13] [36], and it has been used in a 2D numerical study to explain the contribution of changes in cellular size variance to increases in the BSC during a cell death process [21]. The method based on the SFM described here could be a promising tool for assessing disease or monitoring the response to anti-cancer treatment.

\section{REFERENCES}

[1] F. L. Lizzi, M. Ostromogilsky, E. J. Feleppa, M. C. Rorke, and M. M. Yaremko, "Relationship of ultrasonic spectral parameters to features of tissue microstructure", IEEE Trans. Ultrason. Ferroelect. Freq. Contr., vol. 33, no. 3, pp. 319-329, 1986.

[2] M. F. Insana, R. F. Wagner, D. G. Brown and T. J. Hall, "Describing small-scale structure in random media using pulse-echo ultrasound", J. Acoust. Soc. Am., vol. 87, no. 1, pp. 179-192, 1990.

[3] F. L. Lizzi, M. Greenebaum, E. J. Feleppa, and M. Elbaum, "Theoretical framework for spectrum analysis in ultrasonic tissue characterization”, J. Acoust. Soc. Am., vol. 73, no. 4, pp. 1366-1373, 1983.

[4] M. L. Oelze and W. D. O'Brien, "Application of three scattering models to characterization of of solid tumors in mice", Ultrasonic Imaging, vol. 28, no. 2, pp. 83-96, 2006.

[5] E. J. Feleppa, F. L. Lizzi, D. J. Coleman, and M. M. Yaremko, "Diagnostic spectrum analysis in ophthalmology: a physical perspective", Ultrasound Med. Biol., vol. 12, no. 8, pp. 623-631, 1986.

[6] E. J. Feleppa, A. Kalisz, J. B. Sokil-Melgar, F. L. Lizzi, T. Liu, A. L. Rosado, M. C. Shao, W. R. Fair, Y. Wang, M. S. Cookson, V. Reuter and W. D. W. Heston, "Typing of prostate issue by ultrasonic spectrum analysis", IEEE Trans. Ultras. Ferroelectr. Freq. Control., vol. 43, no. 4, pp. 609-619, 1996.

[7] R. C. Waag, J. O. Nilsson, J. P. Astheimer, "Characterization of volume scattering power spectra in isotropic media from power spectra of scattering by planes", J. Acoust. Soc. Am., vol. 74, no. 5, pp. 1555-1571, 1983.

[8] M. L. Oelze, W. D. O’Brien, J. P. Blue, and J. F. Zachary, "Differentiation and characterization of rat mammary fibroadenomas and 4T1 mouse carcinomas using quantitative ultrasound imaging", IEEE Trans. Med. Imaging, vol. 23, no. 6, pp. 764-771, 2004.

[9] A. Han, R. Abuhabsah, J. P. Blue, S. Sarwate and W. D. O’Brien, "Ultrasonic backscatter coefficient quantitative estimates from highconcentration Chinese hamster ovary cell pellet biophantoms", J. Acoust. Soc. Am., vol. 130, no. 6, pp. 4139-4147, 2011. 
[10] J. McNew, R. Lavarello and W. D. O’Brien, "Sound scattering from two concentric fluid spheres (L)”, J. Acoust. Soc. Am., vol. 125, no. 1, pp. 1-4, 2009.

[11] A. Han and W. D. O'Brien, "Structure function for high-concentration biophantoms of polydiperse scatterer sizes", IEEE Trans. Ultras. Ferroelectr. Freq. Control., vol. 62, no. 2, pp. 303-318, 2015.

[12] R. M. Vlad, R. K. Saha, N. M. Alajez, S. Ranieari, G. J. Czarnota and M. C. Kolios, "An increase in cellular size variance contributes to the increase in ultrasound backscatter during cell death", Ultrasound in Medicine \& Biology, vol. 36, no. 9, pp. 1546-1558, 2010.

[13] D. Savery and G. Cloutier, "A point process approach to assess the frequency dependence of ultrasound backscattering by aggregating red blood cells", J. Acoust. Soc. Am., vol. 110, no. 6, pp. 3252-3262, 2001.

[14] V. Twersky, "Low-frequency scattering by correlated distributions of randomly oriented particles", J. Acoust. Soc. Am., vol. 81, no. 5, pp. 1609-1618, 1987.

[15] E. Franceschini and R. Guillermin, "Experimental assessment of four ultrasound scattering models for characterizing concentrated tissuemimicking phantoms", J. Acoust. Soc. Am., vol. 132, no. 6, pp. 3735-3747, 2012.

[16] E. Franceschini, R. Guillermin, F. Tourniaire, S. Roffino, E. Lamy and J.-F. Landrier, "Structure Factor Model for understanding the measured backscatter coefficients from concentrated cell pellet biophantoms", J. Acoust. Soc. Amer., vol. 135, no. 6, pp. 3620-3631, 2014.

[17] E. Franceschini, R. de Monchy and J. Mamou, "Quantitative characterization of tissue microstructure on concentrated cell pellet biophantoms based on the structure factor model", submitted to IEEE Trans. Ultras. Ferroelectr. Freq. Control. special issue on Quantitative UltrasoundBased Tissue Characterization

[18] R. Kuc and M. Schwartz, "Estimating the acoustic attenuation coefficient slope for liver from reflected ultrasound signals", IEEE Trans. Sonics Ultrasonics, vol. SU-26, no. 5, pp. 353-362, 1979.

[19] F. A. Duck, "Physical Properties of Tissue: A Comprehensive Reference Book", (Academic Press, London, 1990), Chap. 4, p. 95.

[20] S. Maruvada, K. K. Shung, S. H. Wang, "High-frequency backscatter and attenuation measurements of selected bovine tissue between 10 and 30 MHz”, Ultrasound Med. Biol., vol. 26, no. 6, pp. 1043-1049, 2000.

[21] R. M. Vlad, S. Brand, A. Giles, M. C. Kolios and G. J. Czarnota. "Quantitative ultrasound characterization of responses to radiotherapy in cancer mouse models", Clin. Cancer Res., vol. 15, no. 6, pp. 2067-2075, 2009.

[22] C. Fournier, S. L. Bridal, A. Coron and P. Laugier,"Optimization of Attenuation Estimations in Reflection for In Vivo Human Dermis Characterization at $20 \mathrm{MHz}$ ", IEEE Trans. ultras. Ferroelectr. Freq. Control., vol. 50, no. 4, pp. 408-418, 2003.

[23] M. O. Donnell and J. G. Miller, "Quantitative broadband ultrasonic backscatter: An approach to nondestructive evaluation in acoustically inhomogeneous materials”, J. Appl. Phys., vol. 52, no. 2, pp. 1056-1065, 1981.

[24] L. X. Yao, J. A. Zagzebski and E. L. Madsen, "Backscatter coefficient measurements using a reference phantom to extract depth-dependent instrumentation factors", Ultrasonic Imaging, vol. 12, pp. 58-70, 1990.

[25] J. J. Faran, "Sound Scattering by Solid Cylinders and Spheres", J. Acoust. Soc. Amer, vol. 23, no. 4, pp. 405-418, 1951.

[26] M. F. Insana and D. G. Brown, "Acoustic scattering theory applied to soft biological tissues", in Ultrasonic Scattering in Biological Tissues, edited by K. K. Shung and G. A. Thieme (CRC, Boca Raton, FL, 1993), Chap.4, pp. 76-124.

[27] M. S. Wertheim, "Exact solution of the Percus-Yevick integral equation for hard spheres", Physical Review Letters, vol. 10, no. 8, pp. 321-323, 1963.

[28] C. Stockhaus, T. Van Den Ingh, J. Rothuizen, and E. Teske, "A multistep approach in the cytologic evaluation of biopsy samples of dogs with hepatic diseases", Vet. Pathol., vol. 41, no. 5, pp. 461-470, 2004.

[29] "Lymphocytic Series. An educational Supplement to morphology critique 96-05-S" prepared by ALQER May 1996. Retrieved from http: //www.cpsa.ab.ca/libraries

[30] R. K. Saha and G. Cloutier, "Monte Carlo study on ultrasound backscattering by three-dimensional distributions of red blood cells", Phys. Rev. E, vol. 78, no. 6, 061919, 2008.

[31] Y. W. Yuan and K. K. Shung, "Ultrasonic backscatter from flowing whole blood. I: Dependence on shear rate and hematocrit", J. Acoust. Soc. Am., vol. 84, no. 1, pp. 52-58, 1998.

[32] A. D. Pawlicki, A. J. Dapore, S. Sarwate and W. D. O'Brien, “Three-dimensional impedance map analysis of rabbit liver", J. Acoust. Soc. Amer., vol. 130, no. 5, pp. EL334-338, 2011.

[33] N. W. Ashcroft and D. C. Langreth, "Structure of binary liquid mixtures. I", Physical Review, vol. 156, no. 3, pp. 685-692, 1967.

[34] M. D. Sherar, M. B. Noss and F. S. Foster, "Ultrasound backscatter microscopy images the internal structure of living tumour spheroids", Nature, vol. 330, no. 3, pp. 493-495, 1987. 
[35] M. F. Insana and T. J. Hall, "Parametric ultrasound imaging from backscatter coefficient measurements: image formation and interpretation", Ultrasonic Imaging, vol. 12, pp. 245-267, 1990.

[36] E. Franceschini, R. K. Saha and G. Cloutier, “Comparison of three scattering models for ultrasound blood characterization”, IEEE Trans. on Ultrason., Ferroelect., Freq. Contr., vol. 60, no. 11, pp. 2321-2334, 2013.

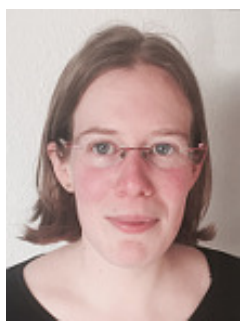

Pauline Muleki-Seya received her M.S. degree in Acoustics from the University of Le Mans in 2011, and her Ph.D. degree in Acoustics from the University of Lyon in 2014. In 2015-2016, she was a Postdoctoral Fellow at the Laboratory of Mechanics and Acoustics in Marseille, conducting research on quantitative ultrasound techniques on soft tissues. Since 2016, she is a Postdoctoral Fellow at the Bioacoustics Research Laboratory from the Department of Electrical and Computer Engineering at the University of Illinois Urbana-Champaign. Her research interests include quantitative ultrasound techniques, ultrasound tissue characterization and sonoporation. She is a member of the French Acoustical Society (SFA).

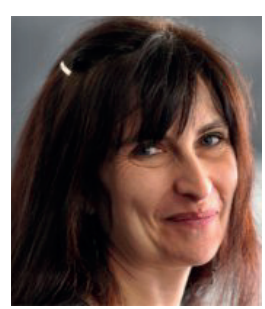

Régine Guillermin was graduated from the Ecole Nationale Supérieure de Physique de Marseille, France, in 1996. She received the Ph.D. degree in physics from the Université de la Méditerranée, Aix-Marseiile II, in 2000. Since 2001, she is a Research Engineer at the French National Center for Scientific Research (CNRS) in the Laboratory of Mechanics and Acoustics CNRS UPR 7051, Marseille. Her work is focused on ultrasonic imaging and ultrasonic quantitative characterization for biomedical and underwater applications. She is a member of the French Acoustical Society (SFA).

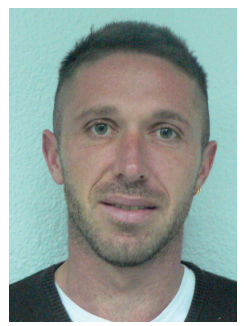

Julien Guglielmi graduated in biological engineering from the Ecoles Pratiques des Hautes Etudes of Paris. Since 2005, he is engineer at the University of Nice Sophia Antipolis and works in the research group of Thierry Pourcher. His research interests include SPECT and ultrasound in vivo imaging.

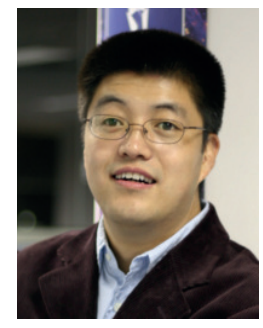

Jiangang Chen was born in Zhangjiakou, Hebei, China, in 1980. He received his B.S. degree in electrical engineering in 2003 from Nanchang Hangkong University, Nanchang, China, M.Phil. degree in biomedical engineering in 2006 from Xian Jiaotong University, Xian, China, and Ph.D. degree in mechanical engineering in 2010 from the Hong Kong Polytechnic University, Hong Kong, China. In 2013, he was a Postdoctoral Fellow at the Ultrasound and Elasticity Imaging Laboratory at Columbia University in New York City. He is currently working as a senior scientist in Philips Research China, Shanghai, China. 


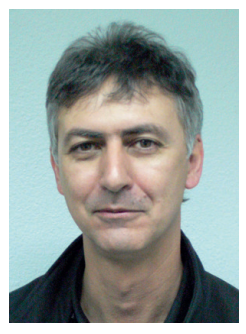

Thierry Pourcher received his Ph.D. degree in molecular biology from the University of Nice Sophia Antipolis, 1989. Since 1990, he is a Researcher at the French Atomic Energy Commission (CEA). He is team leader since 1998. He is the Director of a research laboratory that joints the CEA and the University of Nice since 2004. His research interests include iodide transporters, thyroid metabolism and cancer, in vivo imaging that includes SPECT and ultrasound.

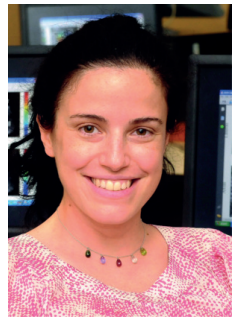

Elisa Konofagou is Professor of Biomedical Engineering and Radiology, and Director of the Ultrasound and Elasticity Imaging Laboratory at Columbia University in New York City. Her main interests are in the development of novel elasticity imaging techniques and therapeutic ultrasound methods and more notably, myocardial elastography, electromechanical and pulse wave imaging, harmonic motion imaging and focused ultrasound therapy and drug delivery in the brain, with several clinical collaborations in the Columbia Presbyterian Medical Center and elsewhere. Elisa is a fellow of the American Institute for Medical and Biological Engineering, member of the IEEE in Engineering in Medicine and Biology Society, IEEE in Ultrasonics, Ferroelectrics and Frequency Control, the Acoustical Society of America and the American Institute of Ultrasound in Medicine. She has co-authored over 140 published articles in the aforementioned fields. She has also served as a technical committee member of the Acoustical Society of America, the International Society of Therapeutic Ultrasound, the IEEE Engineering in Medicine and Biology conference (EMBC), the IEEE International Ultrasonics Symposium and the American Association of Physicists in Medicine (AAPM) as well as a technical standards committee and board of Governors member of the American Institute of Ultrasound in Medicine. Elisa serves as an Associate Editor in the Medical Physics Journal, Ultrasonic Imaging and IEEE Transactions in Ultrasonics, Ferroelectrics and Frequency Control and is recipient of awards from the American Heart Association, the Acoustical Society of America, the American Institute of Ultrasound in Medicine, the Wallace H. Coulter foundation, the National Institutes of Health (NIH), the National Science Foundation (NSF), the Society of Photo-optical Instrumentation Engineers (SPIE) and the Radiological Society of North America (RSNA).

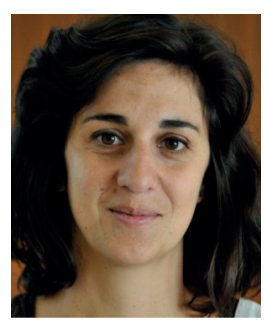

Emilie Franceschini received her M.S. degree in Acoustics from the University of Méditérannée, Marseille, 2003, and the Ph.D. degree in Acoustics from the University of Provence, Marseille, 2006. In 2007, she was a Postdoctoral Fellow at the Laboratory of Biorheology and Medical Ultrasonics, Research Center of the University of Montreal Hospital, Montreal, conducting research in ultrasonic blood characterization. Since October 2008, she is a Researcher at the French National Center for Scientific Research (CNRS) in the Laboratory of Mechanics and Acoustics CNRS UPR 7051, Marseille. Her research interests include ultrasound tissue characterization, ultrasound tomography, quantitative ultrasound techniques and inverse problems. She is a member of the French Acoustical Society (SFA), IEEE and IEEE in Ultrasonics, Ferroelectrics and Frequency Control. 